\title{
Consistent finite-dimensional approximation of phase-field models of fracture
}

\author{
Stefano Almi ${ }^{1}$ (D) Sandro Belz ${ }^{1}$
}

Received: 27 November 2017 / Accepted: 5 December 2018 / Published online: 13 December 2018 (c) Fondazione Annali di Matematica Pura ed Applicata and Springer-Verlag GmbH Germany, part of Springer Nature 2018

\begin{abstract}
In this paper, we focus on the finite-dimensional approximation of quasi-static evolutions of critical points of the phase-field model of brittle fracture. In a space discretized setting, we first discuss an alternating minimization scheme which, together with the usual timediscretization procedure, allows us to construct such finite-dimensional evolutions. Then, passing to the limit as the space discretization becomes finer and finer, we prove that any limit of a sequence of finite-dimensional evolutions is itself a quasi-static evolution of the phase-field model of fracture. Our proof shows for the first time the consistency of a numerical scheme for evolutions of fractures along critical points.
\end{abstract}

Keywords Ambrosio-Tortorelli functional · Phase-field damage · Quasi-static evolution · Critical points $\cdot$ Numerical consistency

Mathematics Subject Classification 49M25 · 49J40

\section{Introduction}

In this paper, we are interested in the study of convergence of numerical schemes for quasistatic evolution of brittle fractures in elastic bodies. We focus on the phase-field (or damage) approximation of fracture studied by Bourdin et al. in $[10,13,14]$, and first introduced by Ambrosio and Tortorelli in [2,3] in the framework of image processing.

In a planar setting, given an open bounded subset $\Omega$ of $\mathbb{R}^{2}$ with Lipschitz boundary $\partial \Omega$, we deal with an energy functional of the form

$$
\mathcal{J}_{\varepsilon}(u, v):=\frac{1}{2} \int_{\Omega}\left(v^{2}+\eta_{\varepsilon}\right)|\nabla u|^{2} \mathrm{~d} x+\kappa \int_{\Omega} \varepsilon|\nabla v|^{2} \mathrm{~d} x+\kappa \int_{\Omega} \frac{(1-v)^{2}}{4 \varepsilon} \mathrm{d} x,
$$

Stefano Almi

stefano.almi@ma.tum.de

Sandro Belz

sandro.belz@ma.tum.de

1 Fakultät für Mathematik, TUM, Boltzmannstr. 3, 85748 Garching bei München, Germany 
where $\varepsilon$ and $\eta_{\varepsilon}$ are two small positive parameters, $u \in H^{1}(\Omega)$ stands for the displacement field, $v \in H^{1}(\Omega ;[0,1])$ denotes the damage variable, and the positive constant $\kappa$ may be interpreted as the toughness of the material, which we assume to be equal to one for the following discussion. From a physical point of view, the variable $v$ in (1.1) takes into account how damaged the elastic body is, so that, for $x \in \Omega, v(x)=0$ means that the damage is complete (fracture) at $x$, while $v(x)=1$ means that the material is perfectly intact at $x$.

In [2,3], it has been shown that choosing $0<\eta_{\varepsilon} \ll \varepsilon$ and letting $\varepsilon \rightarrow 0$, the functional $\mathcal{J}_{\varepsilon}$ $\Gamma$-converges to

$$
\mathcal{G}(u):=\frac{1}{2} \int_{\Omega}|\nabla u|^{2} \mathrm{~d} x+\mathcal{H}^{1}\left(S_{u}\right),
$$

defined on $G S B V(\Omega)$, the space of generalized special function of bounded variation [1]. In (1.2), $\mathcal{H}^{1}$ denotes the 1-dimensional Hausdorff measure and $S_{u}$ stands for the approximate discontinuity set of $u$. In the mathematical model of fracture (see, e.g., [14]), the functional (1.2) represents the energy of an elastic body $\Omega$ subject to an antiplanar displacement $u$ and experiencing the crack $S_{u}$.

In view of such a convergence result, the phase-field functional (1.1) has been successfully used in numerical simulations of crack growth processes (see, for instance, $[6,10,13,14,18$, 19]). The usual strategy consists in constructing finite-dimensional approximations of an evolution by coupling some alternating minimization schemes with Finite Element Methods (FEM). In this framework, we prove the consistency of such schemes by showing their convergence to quasi-static evolution of critical points of $\mathcal{J}_{\mathcal{\varepsilon}}$ as the space discretization becomes finer and finer.

To our knowledge, this paper provides the first consistency proof of a numerical method for such evolutions, going beyond the empirical checks even recently done, for instance, in [7] and related literature. To obtain the result, we needed to fuse classical methods of PDE discretization, such as FEM and their typical quasi-interpolating estimates, together with variational techniques to handle nonlinearities, going far beyond the usual linear setting where, e.g., FEM are employed. Concerning the variational methodology, we innovate over [27], where only variational limits of evolutions along global minimizers were developed and analyzed, essentially, by means of $\Gamma$-convergence techniques (see also $[12,21,22,28,29,35]$ ). Instead, here, perhaps more closely to the work of Braides and coauthors in [16,17], we develop results of consistency for evolutions along critical points, which are more realistic.

We anticipate here that all the results we are going to discuss are still valid in the vectorial case, i.e., when considering the functional

$$
\mathcal{I}_{\varepsilon}(u, v):=\frac{1}{2} \int_{\Omega}\left(v^{2}+\eta_{\varepsilon}\right) \mathbb{C E} u \cdot \mathrm{E} u \mathrm{~d} x+\int_{\Omega} \varepsilon|\nabla v|^{2} \mathrm{~d} x+\int_{\Omega} \frac{(1-v)^{2}}{4 \varepsilon} \mathrm{d} x,
$$

where $u \in H^{1}\left(\Omega ; \mathbb{R}^{2}\right), v \in H^{1}(\Omega)$, and $\mathbb{C}$ is the usual elasticity tensor. For the sake of simplicity, we decided to present here in details only the scalar setting (1.1).

In order to be mathematically more precise, let us briefly describe how we proceed in the approximation of a quasi-static evolution of (1.1) in terms of finite-dimensional evolutions. For notational convenience, we fix the parameters $\varepsilon=\frac{1}{2}$ and $\eta_{\varepsilon}=\eta>0$ and drop the subscript $\varepsilon$ in (1.1), so that we consider the functional

$$
\mathcal{J}(u, v):=\frac{1}{2} \int_{\Omega}\left(v^{2}+\eta\right)|\nabla u|^{2} \mathrm{~d} x+\frac{1}{2} \int_{\Omega}\left(|\nabla v|^{2}+(1-v)^{2}\right) \mathrm{d} x
$$

for $u, v \in H^{1}(\Omega)$. Following the main steps of numerical schemes, we first discretize the function space $H^{1}(\Omega)$ and define the discrete counterpart of the functional $\mathcal{J}$. Given the mesh 
parameter $h>0$, we consider a triangulation $\mathcal{T}_{h}$ of $\Omega$ satisfying the standard requirements arising from interpolation estimates (see (2.14) and [38] for more details). We define the finite-dimensional space

$$
\mathcal{F}_{h}:=\left\{u \in H^{1}(\Omega): u \text { is affine on } K \text { for every } K \in \mathcal{T}_{h}\right\},
$$

and we set, for every $u, v \in \mathcal{F}_{h}$,

$$
\mathcal{J}_{h}(u, v):=\frac{1}{2} \int_{\Omega}\left(\mathrm{P}_{h}\left(v^{2}\right)+\eta\right)|\nabla u|^{2} \mathrm{~d} x+\frac{1}{2} \int_{\Omega}|\nabla v|^{2} \mathrm{~d} x+\frac{1}{2} \int_{\Omega} \mathrm{P}_{h}\left((1-v)^{2}\right) \mathrm{d} x,
$$

where $\mathrm{P}_{h}: C(\bar{\Omega}) \rightarrow \mathcal{F}_{h}$ is the Lagrangian interpolation operator.

In this finite-dimensional framework, we construct a finite-dimensional quasi-static evolution $\left(u_{h}, v_{h}\right)$ driven by the energy functional $\mathcal{J}_{h}$ making use of an alternating minimization algorithm, fusion of the ones developed in $[4,13,32]$. Besides the usual time-discretization procedure, typical in the study of many rate-independent processes [33,34], at each time step we recursively set

$$
\begin{aligned}
u_{j} & :=\arg \min \left\{\mathcal{J}_{h}\left(u, v_{j-1}\right): u \in \mathcal{F}_{h}+\text { boundary conditions }\right\}, \\
v_{j} & :=\arg \min \left\{\mathcal{J}_{h}\left(u_{j}, v\right): v \in \mathcal{F}_{h}, v \leq v_{j-1} \text { in } \Omega\right\} .
\end{aligned}
$$

In the limit as $j \rightarrow \infty$, we compute a critical point of $\mathcal{J}_{h}$. The second step is to define an arc-length parametrization of time based on the distance between two subsequent steps of the minimization scheme (1.5)-(1.6). In the new time variable, we prove that suitable piecewise affine curves interpolating between all the intermediate states of the algorithm (1.5)-(1.6) satisfy an energy balance that we are then able to keep in the time-continuous limit. We refer to Definition 2.8, to Theorem 2.9, and to its proof in Sect. 4 for more details about the minimization scheme and the notion of finite-dimensional evolution.

The last step of our construction is the passage to the limit as the triangulation $\mathcal{T}_{h}$ becomes finer and finer. This is indeed the subject of the proof of Theorem 2.4, where we show that any limit of a sequence of finite-dimensional quasi-static evolutions $\left(u_{h}, v_{h}\right)$ (in non-parametrized time) is a quasi-static evolution in the sense of Definition 2.3, complying with the usual irreversibility and stability conditions and satisfying an energy-dissipation inequality. We remark that in the limit as $h \rightarrow 0$ we are not able to replicate the techniques used in [32] to prove an energy-dissipation balance. The main reason for that is the lack of suitable $h$ independent estimates on the pair $\left(u_{h}, v_{h}\right)$ necessary to guarantee strong convergence in $H^{1} \times H^{1}$ as $h \rightarrow 0$.

In conclusion, we stress once again that the problem of existence of a quasi-static evolution for the phase-field model (1.1) has been already tackled in various papers (see, for instance, $[27,32,36,37])$. In particular, in [32] an existence result of quasi-static evolution for the damage model via critical points of the energy functional (1.1) has been achieved in a space-continuous setting by using an alternate minimization scheme similar to the one described above. In [36], instead, the convergence scheme is based on a local minimization procedure w.r.t. the damage variable $v$. In [27], the evolution problem has been addressed in the setting of global minimizers, giving particular emphasis to the connection between the notions of quasi-static evolution in the phase-field model and in the variational "sharp interface" model of fracture (see, e.g., [26]). Therefore, what we claim to be new in our paper is not the existence result itself, but rather the approximation technique, which shows that the numerical results, obtained through a sort of finite-dimensional implementation of the damage model (1.1) and (1.3), are actually close to the "theoretical" quasi-static evolutions described by Definition 2.3. Moreover, the method we exploit to prove Theorems 2.4 
and 2.9 is suitable for applications and numerical simulations, which we perform in Sect. 6. The purpose of our experiments is not to challenge the efficiency of the numerical methods, but rather to show the consistency of the theoretical result. We keep an extensive numerical analysis for future work.

Plan of the paper The paper is organized as follows: in Sect. 2 we present the evolution problem in full details, giving the definition of quasi-static evolution for the phase-field model (see Definition 2.3) and stating the main result (Theorem 2.4). Then, we start discussing our discretization algorithm and, eventually, in Sect. 3 we present the alternating minimization scheme which is at the core of our approximation. In Sects. 4 and 5, we prove Theorems 2.9 and 2.4, respectively. Finally, in Sect. 6, we present some numerical simulations which exploit the algorithm discussed in this paper.

\section{Setting of the problem}

In this section, we describe the problem setting and introduce the main notation of the paper. We first start with the space-continuous notion, and in the second part of the section, we discuss the space-discrete setting.

\section{Space-continuous setting}

As already mentioned in the Introduction, we are studying quasi-static evolutions in the framework of phase-field approximation of brittle fractures in elastic bodies (for more details see, e.g., $[2,3,25,26])$. Since the aim of this paper is to show a new constructive approach to the evolution problem based on a space discretization procedure, in order to keep the notations as simple as possible we focus here on a two dimensional model. In particular, we consider as a reference configuration the unit square $\Omega:=(0,1)^{2}$ in $\mathbb{R}^{2}$. We believe that this is not a serious restriction and also evolutions in three dimensions can be similarly approached.

Once some $\eta>0$ is fixed, we define the phase-field stored elastic energy as

$$
\mathcal{E}(u, v):=\frac{1}{2} \int_{\Omega}\left(v^{2}+\eta\right)|\nabla u|^{2} \mathrm{~d} x,
$$

where $u \in H^{1}(\Omega)$ denotes the antiplanar displacement and $v \in H^{1}(\Omega)$ stands for the phasefield (or damage) variable. In particular, from (2.1), we deduce that the elastic behavior of $\Omega$ depends pointwise on how damaged the body is, and, due to the presence of the positive parameter $\eta$, the damage is never complete, in the sense that the elastic body $\Omega$ is always able to store a positive amount of elastic energy depending on the displacement $u$. We also recall that the phase-field $v$ is usually constrained to take values in the interval $[0,1]$, where, for $x \in \Omega, v(x)=0$ means that the elastic body $\Omega$ is experiencing a maximal damage in $x$, while $v(x)=1$ means that the material is perfectly sound at $x$. In order to avoid some technical issues related to the discrete setting described in the second part of this section, we simply assume $v$ to belong to $H^{1}(\Omega)$. We will see how the above constraint can be naturally enforced in the space-discrete approximation of the evolution problem. We refer to Proposition 3.1 for more details.

As usual in the phase-field approximation, we add to the stored elastic energy (2.1) a dissipative term $\mathcal{D}(v)$ which depends only on the damage $v \in H^{1}(\Omega)$, namely,

$$
\mathcal{D}(v):=\frac{1}{2} \int_{\Omega}\left(|\nabla v|^{2}+(1-v)^{2}\right) \mathrm{d} x .
$$


In the sense of $\Gamma$-convergence, the dissipation functional $\mathcal{D}$ approximates, in the language of fracture mechanics, the energy dissipated by the crack production, as it has been shown in $[2,3]$.

We are now in a position to introduce the total phase-field energy of the system as the sum of (2.1) and (2.2): for every $u, v \in H^{1}(\Omega)$, we simply set

$$
\mathcal{J}(u, v):=\mathcal{E}(u, v)+\mathcal{D}(v) .
$$

As usual, the evolution problem will be driven by a time-dependent forcing term. Given a time horizon $T>0$, we assume that the elastic body $\Omega$ is subject to a Dirichlet boundary datum $w \in W^{1,2}\left([0, T] ; H^{1}(\Omega)\right)$, so that, for every $t \in[0, T]$, the set of admissible displacement $\mathcal{A}(w(t))$ is defined by

$$
\mathcal{A}(w(t)):=\left\{u \in H^{1}(\Omega): u=w(t) \text { on } \partial \Omega\right\},
$$

where the equality has to be intended in the trace sense. The notation (2.4) will be adopted also for functions $w \in H^{1}(\Omega)$ not depending on time.

In this context, a quasi-static evolution for the damage model is expressed by a pair displacement-damage $(u, v):[0, T] \rightarrow H^{1}(\Omega) \times H^{1}(\Omega ;[0,1])$. The first natural condition we want to impose is the so-called irreversibility of the phase-field variable. Namely, the function $t \mapsto v(t)$ has to be non-increasing. This means that once the elastic body $\Omega$ is damaged, it cannot be repaired, not even partially.

The second property a quasi-static evolution has to satisfy is a stability condition. In our case, to be stable at time $t$ means that the pair $(u(t), v(t))$ is a critical point of the energy (2.3) in the class of pairs $(u, v) \in H^{1}(\Omega) \times H^{1}(\Omega ;[0,1])$ with $u \in \mathcal{A}(w(t))$ and $v \leq v(t)$ a.e. in $\Omega$. Since $\mathcal{J}$ is Fréchet differentiable on $H^{1}(\Omega) \times\left(H^{1}(\Omega) \cap L^{\infty}(\Omega)\right)$ (see [18, Proposition 1.1]) with

$$
\begin{aligned}
\partial_{(u, v)} \mathcal{J}(u, v)[\varphi, \psi]= & \int_{\Omega}\left(v^{2}+\eta\right) \nabla u \cdot \nabla \varphi \mathrm{d} x+\int_{\Omega} v \psi|\nabla u|^{2} \mathrm{~d} x \\
& +\int_{\Omega} \nabla v \cdot \nabla \psi \mathrm{d} x-\int_{\Omega}(1-v) \psi \mathrm{d} x,
\end{aligned}
$$

for every $u \in H^{1}(\Omega), v \in H^{1}(\Omega) \cap L^{\infty}(\Omega), \varphi \in C_{c}^{\infty}(\Omega), \psi \in C^{\infty}(\bar{\Omega})$, the stability condition can be written as

$$
\partial_{(u, v)} \mathcal{J}(u(t), v(t))[\varphi, \psi] \geq 0
$$

for every $\varphi \in C_{c}^{\infty}(\Omega)$ and every $\psi \in C^{\infty}(\bar{\Omega})$ with $\psi \leq 0$.

Remark 2.1 We notice that the inequality in (2.6) and the restriction to test functions $\psi \leq 0$ arise from the irreversibility condition of the damage variable $v(t)$ discussed above.

By the structure of the derivative of $\mathcal{J}$ (2.5), inequality (2.6) can be simply rephrased in terms of the following inequalities:

$$
\begin{aligned}
0 & =\partial_{u} \mathcal{J}(u(t), v(t))[\varphi]=\int_{\Omega}\left(v^{2}(t)+\eta\right) \nabla u(t) \cdot \nabla \varphi \mathrm{d} x \\
0 & \leq \partial_{v} \mathcal{J}(u(t), v(t))[\psi] \\
& =\int_{\Omega} v(t) \psi|\nabla u(t)|^{2} \mathrm{~d} x+\int_{\Omega} \nabla v(t) \cdot \nabla \psi \mathrm{d} x-\int_{\Omega}(1-v(t)) \psi \mathrm{d} x,
\end{aligned}
$$

for every $\varphi \in C_{c}^{\infty}(\Omega)$ and $\psi \in C^{\infty}(\bar{\Omega})$ with $\psi \leq 0$. 
Remark 2.2 The right-hand sides of (2.7) and (2.8) represent the Gateaux derivatives in the direction of $u$ and $v$, respectively.

We also notice that once we know that inequalities (2.7) and (2.8) are satisfied for every test functions $\varphi \in C_{c}^{\infty}(\Omega)$ and $\psi \in C^{\infty}(\bar{\Omega})$ with $\psi \leq 0$ in $\Omega$, by density and truncation argument it is easy to see that they hold also for $\varphi \in H_{0}^{1}(\Omega)$ and $\psi \in H^{1}(\Omega), \psi \leq 0$ a.e. in $\Omega$.

Finally, by the separate convexity of $\mathcal{J}$ w.r.t. the variables $u$ and $v$, from formulas (2.7) and (2.8) we derive the actual stability condition, given in terms of minimum problems: for every $t \in[0, T], u(t)$ minimizes $\mathcal{J}(\cdot, v(t))$ in the class $\mathcal{A}(w(t))$, while $v(t)$ minimizes $\mathcal{J}(u(t), \cdot)$ in the class of functions $v \in H^{1}(\Omega)$ such that $v \leq v(t)$ a.e. in $\Omega$.

This leads us to the following definition of quasi-static evolution for the phase-field model via critical points of the energy $\mathcal{J}$ in (2.1)-(2.3).

Definition 2.3 Let $T>0$ and $w \in W^{1,2}\left([0, T] ; H^{1}(\Omega)\right)$. We say that a pair $(u, v):[0, T] \rightarrow$ $H^{1}(\Omega) \times H^{1}(\Omega)$ is a quasi-static evolution (of critical points) if the following conditions are satisfied:

(1) Irreversibility: $0 \leq v(t) \leq v(\tau) \leq 1$ a.e. in $\Omega$ for every $0 \leq \tau \leq t \leq T$;

(2) Stability: for every $t \in[0, T]$

$$
\begin{aligned}
& \mathcal{J}(u(t), v(t)) \leq \mathcal{J}(u, v(t)) \quad \text { for all } u \in \mathcal{A}(w(t)), \\
& \mathcal{J}(u(t), v(t)) \leq \mathcal{J}(u(t), v) \quad \text { for all } v \in H^{1}(\Omega), v \leq v(t) \text { a.e. in } \Omega \text {. }
\end{aligned}
$$

(3) Energy-dissipation inequality: for every $t \in[0, T]$

$$
\mathcal{J}(u(t), v(t)) \leq \mathcal{J}(u(0), v(0))+\int_{0}^{t} \int_{\Omega}\left(v^{2}(\tau)+\eta\right) \nabla u(\tau) \cdot \nabla \dot{w}(\tau) \mathrm{d} x \mathrm{~d} \tau .
$$

From now on, the dot represents the derivative w.r.t. time $t$.

We can now state the main existence result of the paper, which will be proved in Sect. 5 .

Theorem 2.4 Let $T>0, w \in W^{1,2}\left([0, T] ; H^{1}(\Omega)\right)$, and $u_{0}, v_{0} \in H^{1}(\Omega)$ be such that $u_{0} \in \mathcal{A}(w(0))$ and $0 \leq v_{0} \leq 1$ a.e. in $\Omega$. Assume that the pair $\left(u_{0}, v_{0}\right)$ satisfies the stability conditions at time $t=0$ :

$$
\begin{gathered}
\\
\mathcal{J}\left(u_{0}, v_{0}\right) \leq \mathcal{J}\left(u, v_{0}\right) \quad \text { for all } u \in \mathcal{A}(w(0)), \\
\mathcal{J}\left(u_{0}, v_{0}\right) \leq \mathcal{J}\left(u_{0}, v\right) \quad \text { for all } v \in H^{1}(\Omega), \text { such that } v \leq v_{0} \text { a.e. in } \Omega .
\end{gathered}
$$

Then, there exists a quasi-static evolution $(u, v):[0, T] \rightarrow H^{1}(\Omega) \times H^{1}(\Omega)$ with $u(0)=u_{0}$ and $v(0)=v_{0}$.

Remark 2.5 We again stress that the study of existence of quasi-static evolution for the phasefield model based on the Ambrosio-Tortorelli functional (1.1) is not a novelty. For instance, such a problem has been tackled in $[27,32,36]$ using different convergence schemes, always in a continuous-space setting.

What we claim is the main contribution of our paper is the technique used to construct such an evolution, which is based on the algorithm introduced in [6]. In particular, the method we exploit here is suitable for applications and numerical simulations, since, as we explain in the second part of this section, we first show the existence of a finite-dimensional quasi-static evolution in a discretized $H^{1}$-space (see (2.15)) and then pass to the limit as the discretization becomes finer and finer. We show that in the limit we recover a quasi-static evolution in 
the sense of Definition 2.3. By this argument, we deduce that the numerical scheme used to construct approximate solutions for the evolution problem guarantees convergence to a suitable quasi-static evolution.

\subsection{Space-discrete setting}

Let us now describe the space-discrete counterpart of the above setting. We first want to discretize the domain $\Omega$ following the basic ideas of the finite element method (for more details on the theory see, e.g., [38]). Let us fix $\lambda \in(0,+\infty)$. Given the mesh parameter $h>0$, we consider a triangulation $\mathcal{T}_{h}$ such that $\operatorname{diam}(K) \leq h$ for every $K \in \mathcal{T}_{h}$ and such that

$$
\frac{R_{h}}{\rho_{h}} \leq \lambda \quad \text { uniformly for } h>0,
$$

where $\rho_{h}$ is the minimum of the radii of the incircles of the triangulation $\mathcal{T}_{h}$, and $R_{h}$ is the maximum of the radii of the excircles. The above condition guarantees the usual piecewise affine interpolation estimates. We refer to [38] for more details.

Once we are given the triangulation $\mathcal{T}_{h}$, we need to discretize the function space $H^{1}(\Omega)$. Thus, we define the finite-dimensional function space $\mathcal{F}_{h}$ as the set of continuous functions on $\bar{\Omega}$ that are affine on each triangle $K \in \mathcal{T}_{h}$. More precisely, we set

$$
\mathcal{F}_{h}:=\left\{u \in C(\bar{\Omega}) \cap H^{1}(\Omega): \nabla u \text { is constant a.e. on } K \text { for every } K \in \mathcal{T}_{h}\right\} .
$$

Denoting with $\Delta_{h}$ the set of all the vertices of $\mathcal{T}_{h}$ and setting $N_{h}:=\# \Delta_{h}$, a basis $\left\{\xi_{l}\right\}_{l=1}^{N_{h}}$ of $\mathcal{F}_{h}$ can be defined in the following natural way: for every $l=1, \ldots, N_{h}$, the element $\xi_{l} \in$ $\mathcal{F}_{h}$ is such that

$$
\xi_{l}\left(x_{m}\right)=\delta_{l m} \quad \text { for every } x_{m} \in \Delta_{h},
$$

where $\delta_{l m}$ is the Kronecker delta. We further assume that the basis $\left\{\xi_{l}\right\}_{l=1}^{N_{h}}$ satisfies the stiffness condition

$$
\int_{\Omega} \nabla \xi_{l} \cdot \nabla \xi_{m} \mathrm{~d} x \leq 0 \quad \text { for every } l, m \in\left\{1, \ldots, N_{h}\right\}, l \neq m,
$$

which is fulfilled, e.g., if the angles of the triangles are smaller or equal to $\frac{\pi}{2}$ (see [23]).

Clearly, the space $\mathcal{F}_{h}$ can be endowed with the usual $H^{1}$-norm. In the sequel, we will also use the following:

$$
\|\varphi\|_{\mathcal{F}_{h}}:=\left(\int_{\Omega} \mathrm{P}_{h}\left(\varphi^{2}\right) \mathrm{d} x\right)^{1 / 2} \quad \text { for every } \varphi \in \mathcal{F}_{h},
$$

where $\mathrm{P}_{h}: C(\bar{\Omega}) \rightarrow \mathcal{F}_{h}$ is the Lagrangian interpolant onto the space $\mathcal{F}_{h}$, i.e., the unique operator defined on $C(\bar{\Omega})$ with values in $\mathcal{F}_{h}$ such that

$$
\mathrm{P}_{h}(\varphi)\left(x_{l}\right)=\varphi\left(x_{l}\right) \quad \text { for every } \varphi \in C(\bar{\Omega}) \text { and every } x_{l} \in \Delta_{h} .
$$

It can be easily checked that formula (2.18) defines a norm in $\mathcal{F}_{h}$.

In this framework, we introduce the discrete counterpart of the stored elastic energy (2.1) and of the dissipated energy (2.2): for every $u, v \in \mathcal{F}_{h}$, we set

$$
\begin{aligned}
\mathcal{E}_{h}(u, v) & :=\frac{1}{2} \int_{\Omega}\left(\mathrm{P}_{h}\left(v^{2}\right)+\eta\right)|\nabla u|^{2} \mathrm{~d} x, \\
\mathcal{D}_{h}(v) & :=\frac{1}{2} \int_{\Omega}|\nabla v|^{2} \mathrm{~d} x+\frac{1}{2} \int_{\Omega} \mathrm{P}_{h}\left((1-v)^{2}\right) \mathrm{d} x .
\end{aligned}
$$


As in (2.3), the discrete total energy is the sum of $\mathcal{E}_{h}$ and $\mathcal{D}_{h}$. Hence,

$$
\mathcal{J}_{h}(u, v):=\mathcal{E}_{h}(u, v)+\mathcal{D}_{h}(v) .
$$

We note that, thanks to [38], we can also approximate the Dirichlet boundary datum in $\mathcal{F}_{h}$. More precisely, there exists a sequence $w_{h} \in W^{1,2}\left([0, T] ; \mathcal{F}_{h}\right)$ such that $w_{h} \rightarrow w$ in $W^{1,2}\left([0, T] ; H^{1}(\Omega)\right)$ as $h \rightarrow 0$. In particular, this implies that $w_{h}(t) \rightarrow w(t)$ in $H^{1}(\Omega)$ for every $t \in[0, T]$ and $\dot{w}_{h}(t) \rightarrow \dot{w}(t)$ in $H^{1}(\Omega)$ for a.e. $t \in[0, T]$. Hence, the quasi-static evolution in the space-discrete setting (see Definition 2.6) will be driven by the approximate boundary datum $w_{h}$, and, as in (2.4), for every $h$ and every $t \in[0, T]$ we restrict the set of admissible displacements to

$$
\mathcal{A}_{h}\left(w_{h}(t)\right):=\left\{u \in \mathcal{F}_{h}: u=w_{h}(t) \text { on } \partial \Omega\right\} .
$$

Analogously to Definition 2.3, the notion of finite-dimensional quasi-static evolution reads as follows:

Definition 2.6 Let $T>0$ and $h>0$ be fixed. Let $w_{h} \in W^{1,2}\left([0, T] ; \mathcal{F}_{h}\right)$. We say that a pair of functions $\left(u_{h}, v_{h}\right):[0, T] \rightarrow \mathcal{F}_{h} \times \mathcal{F}_{h}$ is a finite-dimensional quasi-static evolution if it satisfies the following conditions:

(1) Irreversibility: $0 \leq v_{h}(t) \leq v_{h}(\tau) \leq 1$ in $\Omega$ for every $0 \leq \tau \leq t \leq T$;

(2) Stability: for every $t \in(0, T]$ we have

$$
\begin{array}{ll}
\mathcal{J}_{h}\left(u_{h}(t), v_{h}(t)\right) \leq \mathcal{J}_{h}\left(u, v_{h}(t)\right) & \text { for every } u \in \mathcal{A}_{h}\left(w_{h}(t)\right), \\
\mathcal{J}_{h}\left(u_{h}(t), v_{h}(t)\right) \leq \mathcal{J}_{h}\left(u_{h}(t), v\right) & \text { for every } v \in \mathcal{F}_{h}, v \leq v_{h}(t) \text { in } \Omega ;
\end{array}
$$

(3) Energy-dissipation inequality: for every $t \in[0, T]$

$$
\begin{aligned}
\mathcal{J}_{h}\left(u_{h}(t), v_{h}(t)\right) \leq & \mathcal{J}_{h}\left(u_{h}(0), v_{h}(0)\right) \\
& +\int_{0}^{t} \int_{\Omega}\left(\mathrm{P}_{h}\left(v_{h}^{2}(\tau)\right)+\eta\right) \nabla u_{h}(\tau) \cdot \nabla \dot{w}_{h}(\tau) \mathrm{d} x \mathrm{~d} \tau .
\end{aligned}
$$

Remark 2.7 Let us briefly comment on the stability condition (2) of Definition 2.6. As in the space-continuous setting, being the functional $\mathcal{J}_{h}$ separately convex w.r.t. the variables $u$ and $v$, inequalities (2.24) and (2.25) are equivalent to

$$
\begin{aligned}
0 & =\partial_{u} \mathcal{J}_{h}\left(u_{h}(t), v_{h}(t)\right)[\varphi]=\int_{\Omega}\left(\mathrm{P}_{h}\left(v_{h}^{2}(t)\right)+\eta\right) \nabla u_{h}(t) \cdot \nabla \varphi \mathrm{d} x, \\
0 & \leq \partial_{v} \mathcal{J}_{h}\left(u_{h}(t), v_{h}(t)\right)[\psi] \\
& =\int_{\Omega} \mathrm{P}_{h}\left(v_{h}(t) \psi\right)\left|\nabla u_{h}(t)\right|^{2} \mathrm{~d} x+\int_{\Omega} \nabla v_{h}(t) \cdot \nabla \psi \mathrm{d} x-\int_{\Omega} \mathrm{P}_{h}\left(\left(1-v_{h}(t)\right) \psi\right) \mathrm{d} x,
\end{aligned}
$$

for every $\varphi \in \mathcal{A}_{h}(0)$ and $\psi \in \mathcal{F}_{h}$ with $\psi \leq 0$ in $\Omega$.

Moreover, we want property (2) to be satisfied only in the interval $(0, T]$. The motivation of this choice is the following: in Theorem 2.4 (see also Sect. 5) we aim to construct a quasistatic evolution in the space-continuous setting as limit of finite-dimensional quasi-static evolutions. For this reason, as it will be shown in the proof of Theorem 2.4, we need to find ad hoc approximations of the initial conditions $u_{0}, v_{0}$ in the space $\mathcal{F}_{h}$. In doing this, we cannot guarantee to keep track of the stability properties (2.12)-(2.13) of the pair $\left(u_{0}, v_{0}\right)$ (see Theorem 2.4). Therefore, at this stage it is enough for us to have stability for strictly positive time, while in the space-continuous limit we will recover it also for $t=0$. 
In the finite-dimensional case, the existence of a finite-dimensional quasi-static evolution in the sense of Definition 2.6 will actually be a consequence of a stronger result (see Theorem 2.9) which allows us to show, in a suitable time parametrized setting, the validity of an energy-dissipation equality, instead of the simpler inequality stated in (2.26). In order to state precisely the existence result, we need to introduce some further notation.

Following the lines of [32], we introduce two new weighted norms on $\mathcal{F}_{h}$, which could be referred to as energy norms, in the sense that they resemble the energy functional $\mathcal{J}_{h}$ in (2.22): for every $u, v \in \mathcal{F}_{h}$ and every $\varphi \in \mathcal{A}_{h}(0)$ we set

$$
\begin{gathered}
\|\varphi\|_{h, v}^{2}:=\int_{\Omega}\left(\mathrm{P}_{h}\left(v^{2}\right)+\eta\right)|\nabla \varphi|^{2} \mathrm{~d} x \\
\|v\|_{h, u}^{2}:=\int_{\Omega} \mathrm{P}_{h}\left(v^{2}\right)|\nabla u|^{2} \mathrm{~d} x+\int_{\Omega}|\nabla v|^{2} \mathrm{~d} x+\int_{\Omega} \mathrm{P}_{h}\left(v^{2}\right) \mathrm{d} x .
\end{gathered}
$$

We notice that $\|\cdot\|_{h, v}$ in (2.27), which is a norm on $\mathcal{A}_{h}(0)$, actually fails to be a norm on the whole $\mathcal{F}_{h}$. Furthermore, we denote by $\langle\cdot, \cdot\rangle_{h, v}$ and by $\langle\cdot, \cdot\rangle_{h, u}$ the scalar products inducing the norms (2.27) and (2.28), respectively. We refer to "Appendix A" for more properties of $\|\cdot\|_{h, v}$ and $\|\cdot\|_{h, u}$.

Finally, we introduce the slope functions: for every $u, v \in \mathcal{F}_{h}$ we define

$$
\begin{aligned}
\left|\partial_{u} \mathcal{J}_{h}\right|(u, v) & :=\max \left\{-\partial_{u} \mathcal{J}_{h}(u, v)[\varphi]: \varphi \in \mathcal{A}_{h}(0),\|\varphi\|_{h, v} \leq 1\right\}, \\
\left|\partial_{v} \mathcal{J}_{h}\right|(u, v) & :=\max \left\{-\partial_{v} \mathcal{J}_{h}(u, v)[\psi]: \psi \in \mathcal{F}_{h}, \psi \leq 0,\|\psi\|_{h, u} \leq 1\right\} .
\end{aligned}
$$

We notice that the sign restriction in (2.30) is again due to the irreversibility condition on the phase-field variable. We refer to "Appendix A" for some properties of the slopes (2.29)-(2.30).

With this notation, we can give the definition of parametrized finite-dimensional quasistatic evolution.

Definition 2.8 Let $T>0$ and $h>0$ be fixed. Let $w_{h} \in W^{1,2}\left([0, T] ; \mathcal{F}_{h}\right)$. We say that a triple $\left(t_{h}, u_{h}, v_{h}\right):[0, S] \rightarrow[0, T] \times \mathcal{F}_{h} \times \mathcal{F}_{h}, S \in(0,+\infty)$, is a parametrized finite-dimensional quasi-static evolution if it satisfies the following conditions:

(1) Time regularity: $t_{h} \in W^{1, \infty}([0, S] ;[0, T]), u_{h} \in W^{1,2}\left([0, S] ; \mathcal{F}_{h}\right)$, and $v_{h} \in$ $W^{1, \infty}\left([0, S] ; \mathcal{F}_{h}\right)$

(2) Time parametrization: $0 \leq t_{h}(s) \leq t_{h}(\sigma) \leq T$ for every $0 \leq s \leq \sigma \leq S$ and $t_{h}(0)=0$, $t_{h}(S)=T$

(3) Irreversibility: $0 \leq v_{h}(\sigma) \leq v_{h}(s) \leq 1$ in $\Omega$ for every $0 \leq s \leq \sigma \leq S$;

(4) Stability: for every $s \in(0, S]$ such that $t_{h}^{\prime}(s)>0$ we have

$$
\begin{array}{ll}
\mathcal{J}_{h}\left(u_{h}(s), v_{h}(s)\right) \leq \mathcal{J}_{h}\left(u, v_{h}(s)\right) & \text { for every } u \in \mathcal{A}_{h}\left(w_{h}\left(t_{h}(s)\right)\right), \\
\mathcal{J}_{h}\left(u_{h}(s), v_{h}(s)\right) \leq \mathcal{J}_{h}\left(u_{h}(s), v\right) & \text { for every } v \in \mathcal{F}_{h}, v \leq v_{h}(s) \text { in } \Omega ;
\end{array}
$$

(5) Energy-dissipation equality: for every $s \in[0, S]$

$$
\begin{aligned}
\mathcal{J}_{h}\left(u_{h}(s), v_{h}(s)\right)= & \mathcal{J}_{h}\left(u_{h}(0), v_{h}(0)\right)-\int_{0}^{s}\left|\partial_{u} \mathcal{J}_{h}\right|\left(u_{h}(\sigma), v_{h}(\sigma)\right)\left\|u_{h}^{\prime}(\sigma)\right\|_{h, v_{h}(\sigma)} \mathrm{d} \sigma \\
& -\int_{0}^{s}\left|\partial_{v} \mathcal{J}_{h}\right|\left(u_{h}(\sigma), v_{h}(\sigma)\right)\left\|v_{h}^{\prime}(\sigma)\right\|_{h, u_{h}(\sigma)} \mathrm{d} \sigma \\
& +\int_{0}^{s} \int_{\Omega}\left(\mathrm{P}_{h}\left(v_{h}^{2}(\sigma)\right)+\eta\right) \nabla u_{h}(\sigma) \cdot \nabla \dot{w}_{h}\left(t_{h}(\sigma)\right) t_{h}^{\prime}(\sigma) \mathrm{d} x \mathrm{~d} \sigma
\end{aligned}
$$


From now on, the symbol' denotes the derivative w.r.t. $s$.

We state here the main existence result for the finite-dimensional setting. Before showing the proof, we need some auxiliary results. Here, we only mention that the construction of a parametrized finite-dimensional quasi-static evolution is based on the incremental procedure described in Sect. 3 and presented in a different context in [4,32].

Theorem 2.9 Let $h>0, w_{h} \in W^{1,2}\left([0, T] ; \mathcal{F}_{h}\right)$, and $u_{0, h}, v_{0, h} \in \mathcal{F}_{h}$ be such that $u_{0, h} \in \mathcal{A}_{h}\left(w_{h}(0)\right)$ and $0 \leq v_{0, h} \leq 1$ in $\Omega$. Then, there exists a parametrized finitedimensional quasi-static evolution $\left(t_{h}, u_{h}, v_{h}\right):[0, S] \rightarrow[0, T] \times \mathcal{F}_{h} \times \mathcal{F}_{h}, S \in(0,+\infty)$, with $\left(u_{h}(0), v_{h}(0)\right)=\left(u_{0, h}, v_{0, h}\right)$.

As a direct consequence of Theorem 2.9, we get the existence of a finite-dimensional quasi-static evolution in the sense of Definition 2.6.

Corollary 2.10 Let $h>0, w_{h} \in W^{1,2}\left([0, T] ; \mathcal{F}_{h}\right)$, and $u_{0, h}, v_{0, h} \in \mathcal{F}_{h}$ be such that $u_{0, h} \in$ $\mathcal{A}_{h}\left(w_{h}(0)\right)$ and $0 \leq v_{0, h} \leq 1$ in $\Omega$. Then, there exists a finite-dimensional quasi-static evolution $\left(u_{h}, v_{h}\right):[0, T] \rightarrow \mathcal{F}_{h} \times \mathcal{F}_{h}$ such that $\left(u_{h}(0), v_{h}(0)\right)=\left(u_{0, h}, v_{0, h}\right)$.

Proof Let $w_{h}, u_{0, h}$, and $v_{0, h}$ be as in the statement of the corollary, and let $\left(t_{h}, \tilde{u}_{h}, \tilde{v}_{h}\right):[0, S]$ $\rightarrow[0, T] \times \mathcal{F}_{h} \times \mathcal{F}_{h}, S \in(0,+\infty)$, be a parametrized finite-dimensional quasi-static evolution with initial conditions $\left(\tilde{u}_{h}(0), \tilde{v}_{h}(0)\right)=\left(u_{0, h}, v_{0, h}\right)$. For every $t \in[0, T]$, we set

$$
s(t):=\min \left\{s \in[0, S]: t_{h}(s)=t\right\} \text { and }\left(u_{h}(t), v_{h}(t)\right):=\left(\tilde{u}_{h}(s(t)), \tilde{v}_{h}(s(t))\right) .
$$

Clearly, $v_{h}$ satisfies condition (1) of Definition 2.6. The energy-dissipation inequality (2.26) follows by the simple inequality

$$
\begin{aligned}
\mathcal{J}_{h}\left(\tilde{u}_{h}(s(t)), \tilde{v}_{h}(s(t))\right) \leq & \mathcal{J}_{h}\left(u_{0, h}, v_{0, h}\right) \\
& +\int_{0}^{s(t)} \int_{\Omega}\left(\mathrm{P}_{h}\left(\tilde{v}_{h}^{2}(\sigma)\right)+\eta\right) \nabla \tilde{u}_{h}(\sigma) \cdot \nabla \dot{w}_{h}(\sigma) t_{h}^{\prime}(\sigma) \mathrm{d} x \mathrm{~d} \sigma
\end{aligned}
$$

and by the change of variable $\tau=t_{h}(\sigma)$ in the last integral.

Finally, in order to deduce (2.24)-(2.25) from (2.31)-(2.32), we distinguish two cases: $t_{h}^{\prime}(s(t))>0$ and $t_{h}^{\prime}(s(t))=0$. In the former case, (2.24)-(2.25) and (2.31)-(2.32) coincide. In the latter case, we know that, by definition of $s(t)$, there exists a sequence $\sigma_{k} \in[0, S]$ such that $\sigma_{k} \nearrow s(t)$ with $t_{h}^{\prime}\left(\sigma_{k}\right)>0$. Noticing that $\tilde{u}_{h}\left(\sigma_{k}\right) \rightarrow u_{h}(t)$ in $\mathcal{F}_{h}$, that $\tilde{v}_{h}\left(\sigma_{k}\right) \rightarrow v_{h}(t)$ in $\mathcal{F}_{h}$, and that $\tilde{v}_{h}\left(\sigma_{k}\right) \geq v_{h}(t)$ for every $k$, applying Lemma 3.3 below we deduce (2.24)(2.25).

Remark 2.11 We stress here that we are not able to prove, passing to the limit as the mesh parameter $h$ tends to 0 , that a sequence of parametrized finite-dimensional quasi-static evolutions converges to some parametric quasi-static evolution in the space-continuous setting. In particular, we are not able to guarantee the right $h$-independent estimates on $u_{h}$ and $v_{h}$ that would allow us to show an energy-dissipation equality similar to (2.33) for a spacecontinuous limit $(u, v)$. For this reason, in Sect. 5, we will only be able to show that any limit as $h \rightarrow 0$ of a sequence of finite-dimensional quasi-static evolution is itself a quasi-static evolution in the sense of Definition 2.3.

We refer the interested reader to [32] for more technical details concerning the proof of existence of a parametrized quasi-static evolution in the pure space-continuous setting, regardless of the approximability of such an evolution through space-discrete ones. 


\section{Notation}

From now on, we will denote by $\|\cdot\|_{H^{1}}$ the $H^{1}$-norm and by $\|\cdot\|_{p}$ the usual $L^{p}$-norm for $p \in[1,+\infty]$.

\section{The alternate minimization scheme}

In this section, we describe the core of our convergence algorithm, which will allow us to construct critical points of the energy functional $\mathcal{J}_{h}$ satisfying proper boundary and irreversibility conditions. Such a scheme will be exploited in the proof of Theorem 2.9. More precisely, for a given mesh parameter $h>0$, we fix two functions $v_{0}, w \in \mathcal{F}_{h}$, with $v_{0} \geq 0$ in $\Omega$ and we show a constructive way to find a critical point $(\bar{u}, \bar{v}) \in \mathcal{F}_{h} \times \mathcal{F}_{h}$ of the functional $\mathcal{J}_{h}$ under the constraints $\bar{u}=w$ on $\partial \Omega$ and $\bar{v} \leq v_{0}$ in $\Omega$.

The recursive scheme we adopt here is a modification of the strategy presented in [6] in a finite-dimensional setting, and similar to the alternate minimization procedure used in [32] for the continuous phase-field model. What we have to take care of in our context is the presence of the operator $\mathrm{P}_{h}$ of (2.19) in the definition of the functional $\mathcal{J}_{h}$ (see (2.20)-(2.22)).

For every $j \in \mathbb{N} \backslash\{0\}$, we define the functions $u_{j}$ and $v_{j}$ in $\mathcal{F}_{h}$ as follows:

$$
\begin{gathered}
u_{j}:=\arg \min \left\{\mathcal{J}_{h}\left(u, v_{j-1}\right): u \in \mathcal{A}_{h}(w)\right\}, \\
v_{j}:=\arg \min \left\{\mathcal{J}_{h}\left(u_{j}, v\right): v \in \mathcal{F}_{h}, v \leq v_{j-1} \text { in } \Omega\right\} .
\end{gathered}
$$

The existence of minimizers of (3.1) is standard. The uniqueness follows by the strict convexity of the functional $\mathcal{J}_{h}(\cdot, v)$ for $v \in \mathcal{F}_{h}$.

In the following proposition, we briefly discuss the existence and uniqueness of $v_{j}$. We also show the usual bound $0 \leq v_{j} \leq 1$, which does not follow by simple truncation argument because of the nature of the function space $\mathcal{F}_{h}$ and of the presence of the interpolation operator $\mathrm{P}_{h}: C(\bar{\Omega}) \rightarrow \mathcal{F}_{h}$. The proof is contained in the "Appendix A".

Proposition 3.1 The minimum problem (3.2) admits a unique solution. Moreover, the solution $v_{j} \in \mathcal{F}_{h}$ satisfies $0 \leq v_{j} \leq 1$ for every $j \in \mathbb{N} \backslash\{0\}$.

Proof See "Appendix A".

In the following lemma, we show a "one-step" energy balance involving the pairs $\left(u_{j}, v_{j}\right)$ and $\left(u_{j+1}, v_{j+1}\right)$ constructed in (3.1)-(3.2).

Lemma 3.2 Let $j \in \mathbb{N}$. For every $r \in[0,1]$ let

$$
u(r):=(1-r) u_{j}+r u_{j+1} \quad \text { and } \quad v(r):=(1-r) v_{j}+r v_{j+1} .
$$

Then, for every $\bar{r} \in[0,1]$, the following equalities hold:

$$
\begin{aligned}
\mathcal{J}_{h}\left(u(\bar{r}), v_{j}\right) & =\mathcal{J}_{h}\left(u_{j}, v_{j}\right)-\int_{0}^{\bar{r}}\left|\partial_{u} \mathcal{J}_{h}\right|\left(u(r), v_{j}\right)\left\|u^{\prime}(r)\right\|_{h, v_{j}} \mathrm{~d} r, \\
\mathcal{J}_{h}\left(u_{j+1}, v(\bar{r})\right) & =\mathcal{J}_{h}\left(u_{j+1}, v_{j}\right)-\int_{0}^{\bar{r}}\left|\partial_{v} \mathcal{J}_{h}\right|\left(u_{j+1}, v(r)\right)\left\|v^{\prime}(r)\right\|_{h, u_{j+1}} \mathrm{~d} r,
\end{aligned}
$$

where' denotes the derivative w.r.t. $r$. 
Proof By the minimality of $u_{j+1}$, for every $\varphi \in \mathcal{A}_{h}(0)$ we have

$$
\begin{aligned}
\partial_{u} \mathcal{J}_{h}\left(u_{j}, v_{j}\right)[\varphi] & =\partial_{u} \mathcal{J}_{h}\left(u_{j+1}, v_{j}\right)[\varphi]+\int_{\Omega}\left(\mathrm{P}_{h}\left(v_{j}^{2}\right)+\eta\right) \nabla\left(u_{j}-u_{j+1}\right) \cdot \nabla \varphi \mathrm{d} x \\
& =\int_{\Omega}\left(\mathrm{P}_{h}\left(v_{j}^{2}\right)+\eta\right) \nabla\left(u_{j}-u_{j+1}\right) \cdot \nabla \varphi \mathrm{d} x=\left\langle u_{j}-u_{j+1}, \varphi\right\rangle_{h, v_{j}} .
\end{aligned}
$$

Hence, by definition of the slope (2.29), we deduce that

$$
\left|\partial_{u} \mathcal{J}_{h}\right|\left(u_{j}, v_{j}\right)=-\partial_{u} \mathcal{J}_{h}\left(u_{j}, v_{j}\right) \frac{\left[u_{j+1}-u_{j}\right]}{\left\|u_{j+1}-u_{j}\right\|_{h, v_{j}}} .
$$

Let us now fix $r \in(0,1]$. By linearity, for every $\varphi \in \mathcal{A}_{h}(0)$ we have

$$
\begin{aligned}
\partial_{u} \mathcal{J}_{h}\left(u(r), v_{j}\right)[\varphi] & =r \partial_{u} \mathcal{J}_{h}\left(u_{j+1}, v_{j}\right)[\varphi]+(1-r) \partial_{u} \mathcal{J}_{h}\left(u_{j}, v_{j}\right)[\varphi] \\
& =(1-r) \partial_{u} \mathcal{J}_{h}\left(u_{j}, v_{j}\right)[\varphi] .
\end{aligned}
$$

In view of (3.5), we get that

$$
\left|\partial_{u} \mathcal{J}_{h}\right|\left(u(r), v_{j}\right)\left\|u^{\prime}(r)\right\|_{h, v_{j}}=-\partial_{u} \mathcal{J}_{h}\left(u(r), v_{j}\right)\left[u^{\prime}(r)\right] .
$$

Combining the chain-rule and (3.6), we obtain (3.3).

As for (3.4), for every $\varphi \in \mathcal{F}_{h}$ with $\varphi \leq 0$ we have

$$
\begin{aligned}
\partial_{v} \mathcal{J}_{h}\left(u_{j+1}, v_{j}\right)[\varphi]= & \partial_{v} \mathcal{J}_{h}\left(u_{j+1}, v_{j+1}\right)[\varphi]+\int_{\Omega} \mathrm{P}_{h}\left(\left(v_{j}-v_{j+1}\right) \varphi\right)\left|\nabla u_{j+1}\right|^{2} \mathrm{~d} x \\
& +\int_{\Omega} \nabla\left(v_{j}-v_{j+1}\right) \cdot \nabla \varphi \mathrm{d} x+\int_{\Omega} \mathrm{P}_{h}\left(\left(v_{j}-v_{j+1}\right) \varphi\right) \mathrm{d} x \\
= & \partial_{v} \mathcal{J}_{h}\left(u_{j+1}, v_{j+1}\right)[\varphi]+\left\langle v_{j}-v_{j+1}, \varphi\right\rangle_{h, u_{j+1}} .
\end{aligned}
$$

Since, by definition of $v_{j+1}$ in (3.2),

$$
\begin{aligned}
& \partial_{v} \mathcal{J}_{h}\left(u_{j+1}, v_{j+1}\right)[\varphi] \geq 0 \quad \text { for every } \varphi \in \mathcal{F}_{h}, \varphi \leq 0 \text { in } \Omega, \\
& \partial_{v} \mathcal{J}_{h}\left(u_{j+1}, v_{j+1}\right)\left[v_{j+1}-v_{j}\right]=0,
\end{aligned}
$$

inequality (3.7) implies that

$$
\left|\partial_{v} \mathcal{J}_{h}\right|\left(u_{j+1}, v_{j}\right)=-\partial_{v} \mathcal{J}_{h}\left(u_{j+1}, v_{j}\right) \frac{\left[v_{j+1}-v_{j}\right]}{\left\|v_{j+1}-v_{j}\right\|_{h, u_{j+1}}} .
$$

For $r \in(0,1]$, again by linearity we can write

$$
\partial_{v} \mathcal{J}_{h}\left(u_{j+1}, v(r)\right)[\varphi]=(1-r) \partial_{v} \mathcal{J}_{h}\left(u_{j+1}, v_{j}\right)[\varphi]+r \partial_{v} \mathcal{J}_{h}\left(u_{j+1}, v_{j+1}\right)[\varphi],
$$

which implies, together with (3.8), that

$$
\left|\partial_{v} \mathcal{J}_{h}\right|\left(u_{j+1}, v(r)\right)\left\|v^{\prime}(r)\right\|_{h, u_{j+1}}=-\partial_{v} \mathcal{J}_{h}\left(u_{j+1}, v(r)\right)\left[v^{\prime}(r)\right] .
$$

Again, combining the chain-rule and (3.9) we get (3.4), and the proof is thus concluded.

We now want to show that any limit $(\bar{u}, \bar{v})$ of the sequence $\left(u_{j}, v_{j}\right)$ defined in (3.1)-(3.2) is a critical point of the functional $\mathcal{J}_{h}$ satisfying $\bar{u} \in \mathcal{A}_{h}(w)$ and $\bar{v} \leq v_{0}$ in $\Omega$. To do so, we first show a stability property of the minimum problems (3.1) and (3.2). This is the aim of the following lemma, which is stated in a more general setting than the one needed in this section, since it will be useful also in the proof of Theorem 2.9. 
Lemma 3.3 Let $u_{k}, v_{k}, w_{k}, z_{k} \in \mathcal{F}_{h}$ be such that $u_{k} \in \mathcal{A}_{h}\left(w_{k}\right)$ and

$$
\begin{gathered}
\mathcal{J}_{h}\left(u_{k}, z_{k}\right) \leq \mathcal{J}_{h}\left(u, z_{k}\right) \quad \text { for every } u \in \mathcal{A}_{h}\left(w_{k}\right), \\
\mathcal{J}_{h}\left(u_{k}, v_{k}\right) \leq \mathcal{J}_{h}\left(u_{k}, v\right) \quad \text { for every } v \in \mathcal{F}_{h} \text { such that } v \leq z_{k} \text { in } \Omega .
\end{gathered}
$$

Assume that there exist $\bar{u}, \bar{v}, \bar{w}, \bar{z} \in \mathcal{F}_{h}$ such that $u_{k} \rightarrow \bar{u}, v_{k} \rightarrow \bar{v}, w_{k} \rightarrow \bar{w}$, and $z_{k} \rightarrow \bar{z}$ in $\mathcal{F}_{h}$ as $k \rightarrow+\infty$. Then $\bar{u} \in \mathcal{A}_{h}(\bar{w})$ and

$$
\begin{gathered}
\mathcal{J}_{h}(\bar{u}, \bar{z}) \leq \mathcal{J}_{h}(u, \bar{z}) \quad \text { for every } u \in \mathcal{A}_{h}(\bar{w}), \\
\mathcal{J}_{h}(\bar{u}, \bar{v}) \leq \mathcal{J}_{h}(\bar{u}, v) \quad \text { for every } v \in \mathcal{F}_{h} \text { such that } v \leq \bar{z} \text { in } \Omega .
\end{gathered}
$$

Proof Let us prove (3.12). For every $k \in \mathbb{N}$ and every $u \in \mathcal{A}_{h}(\bar{w})$, we have

$$
\mathcal{J}_{h}\left(u_{k}, z_{k}\right) \leq \mathcal{J}_{h}\left(u+w_{k}-\bar{w}, z_{k}\right) .
$$

Since $u+w_{k}-\bar{w} \rightarrow u$ in $\mathcal{F}_{h}$ as $k \rightarrow+\infty$, passing to the limit in (3.14) we get (3.12).

As for (3.13), for every $v \in \mathcal{F}_{h}$ such that $v \leq \bar{z}$ in $\Omega$ we have that $z_{k}+v-\bar{z} \leq z_{k}$. Hence, by $(3.11)$,

$$
\mathcal{J}_{h}\left(u_{k}, v_{k}\right) \leq \mathcal{J}_{h}\left(u_{k}, z_{k}+v-\bar{z}\right) .
$$

Passing to the limit in the previous inequality we get (3.13).

We are now ready to show the convergence of the sequence $\left(u_{j}, v_{j}\right)$, defined by (3.1) and (3.2), to a critical point of $\mathcal{J}_{h}$.

Proposition 3.4 Let $v_{0}, w \in \mathcal{F}_{h}$ with $v_{0} \geq 0$, and let $u_{j}, v_{j}$ be defined by (3.1) and (3.2), respectively. Then the following facts hold:

(1) there exist $\bar{u}, \bar{v} \in \mathcal{F}_{h}$ such that $u_{j} \rightarrow \bar{u}$ and $v_{j} \rightarrow \bar{v}$ in $\mathcal{F}_{h}$ as $j \rightarrow+\infty$;

(2) the limit function $\bar{v}$ satisfies $0 \leq \bar{v} \leq 1$;

(3) the limit functions $\bar{u}, \bar{v} \in \mathcal{F}_{h}$ satisfy

$$
\begin{array}{ll}
\mathcal{J}_{h}(\bar{u}, \bar{v}) \leq \mathcal{J}_{h}(u, \bar{v}) & \text { for every } u \in \mathcal{A}_{h}(w), \\
\mathcal{J}_{h}(\bar{u}, \bar{v}) \leq \mathcal{J}_{h}(\bar{u}, v) & \text { for every } v \in \mathcal{F}_{h} \text { with } v \leq \bar{v} .
\end{array}
$$

Proof By definition of $u_{j}$ and $v_{j}$, for every $j \geq 2$ we have

$$
\mathcal{J}_{h}\left(u_{j}, v_{j}\right) \leq \mathcal{J}_{h}\left(u_{j}, v_{j-1}\right) \leq \mathcal{J}_{h}\left(u_{j-1}, v_{j-1}\right) .
$$

Iterating inequality (3.17), we obtain

$$
\mathcal{J}_{h}\left(u_{j}, v_{j}\right) \leq \mathcal{J}_{h}\left(u_{1}, v_{1}\right) \leq \mathcal{J}_{h}\left(w, v_{0}\right)<+\infty,
$$

from which we deduce that the sequences $u_{j}$ and $v_{j}$ are bounded in $\mathcal{F}_{h}$. Being $v_{j}$ a decreasing sequence with values in the interval $[0,1]$ and being $\mathcal{F}_{h}$ finite-dimensional, we deduce that there exists $\bar{v} \in \mathcal{F}_{h}$ such that $v_{j} \rightarrow \bar{v}$ in $\mathcal{F}_{h}$ and $0 \leq \bar{v} \leq 1$ in $\Omega$, so that property (2) holds. Moreover, by compactness, there exists $\bar{u} \in \mathcal{F}_{h}$ such that, up to a subsequence, $u_{j} \rightarrow \bar{u}$.

Property (3) results from Lemma 3.3 applied to the sequences $u_{j}, v_{j}$, with fixed boundary datum $w \in \mathcal{F}_{h}$. By uniqueness of solution to (3.15), we also deduce that the whole sequence $u_{j}$ converges to $\bar{u}$ in $\mathcal{F}_{h}$, and the proof is thus concluded.

We conclude this section proving a continuity property of the minimum problem (3.1) w.r.t. the phase-field $v$ and the boundary data $w$. This result will be useful in the construction of a suitable time parametrization for the discrete solutions (see Sect. 4). 
Proposition 3.5 Let $M>0$ and $u_{1}, u_{2}, v_{1}, v_{2}, w_{1}, w_{2} \in \mathcal{F}_{h}$ be such that, for $i=1,2$, $\left\|v_{i}\right\|_{\infty} \leq M$ and

$$
u_{i}=\arg \min \left\{\mathcal{J}_{h}\left(u, v_{i}\right): u \in \mathcal{A}_{h}\left(w_{i}\right)\right\} .
$$

Then, there exists a positive constant $C=C\left(M, w_{i}, h\right)$ such that

$$
\left\|u_{1}-u_{2}\right\|_{H^{1}} \leq C\left(\left\|w_{1}-w_{2}\right\|_{H^{1}}+\left\|v_{1}-v_{2}\right\|_{\mathcal{F}_{h}}\right) .
$$

Proof Let us consider $u_{*}=\arg \min \left\{\mathcal{J}_{h}\left(u, v_{1}\right): u \in \mathcal{A}_{h}\left(w_{2}\right)\right\}$. Then, it is easy to see that there exists $C>0$ such that

$$
\left\|u_{1}-u_{*}\right\|_{H^{1}} \leq C\left\|w_{1}-w_{2}\right\|_{H^{1}} .
$$

In order to estimate $\left\|u_{*}-u_{2}\right\|_{H^{1}}$, for every $\varphi \in \mathcal{A}_{h}(0)$ we write

$$
\int_{\Omega}\left(\mathrm{P}_{h}\left(v_{1}^{2}\right)+\eta\right) \nabla\left(u_{2}-u_{*}\right) \cdot \nabla \varphi \mathrm{d} x=\int_{\Omega} \mathrm{P}_{h}\left(v_{1}^{2}-v_{2}^{2}\right) \nabla u_{2} \cdot \nabla \varphi \mathrm{d} x .
$$

Testing $\varphi=u_{2}-u_{*}$ in (3.19) and recalling the hypothesis $\left\|v_{i}\right\|_{\infty} \leq M$, we deduce that

$$
\eta\left\|u_{2}-u_{*}\right\|_{H^{1}}^{2} \leq 2 M \int_{\Omega}\left|\mathrm{P}_{h}\left(v_{1}-v_{2}\right)\right|\left|\nabla u_{2}\right|\left|\nabla\left(u_{2}-u_{*}\right)\right| \mathrm{d} x .
$$

From the previous inequality, we deduce (3.18) by Hölder inequality, using the fact that $\mathcal{F}_{h}$ is finite dimensional, so that all the norms are equivalent, and $\left\|u_{2}\right\|_{H^{1}} \leq c\left\|w_{2}\right\|_{H^{1}}$ for some positive $c$. The dependence of $C$ in (3.18) from the mesh parameter $h$ is due to the $h$-dependent equivalence of norms in $\mathcal{F}_{h}$.

\section{Construction of parametrized finite-dimensional quasi-static evolutions}

We are now ready to prove Theorem 2.9, that is, the existence of a parametrized finitedimensional quasi-static evolution in the sense of Definition 2.8. The strategy of the proof is based on a time-discretization procedure, typical of many rate-independent processes (see, e.g., $[33,34])$ and on an arc-length reparametrization of time similar to the one used in [32].

Let $h>0$. For every $k \in \mathbb{N}$, we consider the uniform subdivision of the time interval $[0, T]$ given by $t_{i}^{k}:=\frac{i T}{k}, i=0, \ldots, k$. In order to construct a discrete in time evolution in the finitedimensional setting we follow the algorithm proposed in [6]: for $i=0$ we set $u_{0}^{k, h}:=u_{0, h}$ and $v_{0}^{k, h}:=v_{0, h}$. For $i \geq 1$, at the instant $t_{i}^{k}$, we construct a critical point $\left(u_{i}^{k, h}, v_{i}^{k, h}\right) \in \mathcal{F}_{h} \times \mathcal{F}_{h}$ of $\mathcal{J}_{h}$ as limit of the alternating minimization process described in Sect. 3. More precisely, let us set

$$
u_{i, 0}^{k, h}:=u_{i-1}^{k, h}+w_{h}\left(t_{i}^{k}\right)-w_{h}\left(t_{i-1}^{k}\right) \quad \text { and } \quad v_{i, 0}^{k, h}:=v_{i-1}^{k, h} .
$$

For $j \in \mathbb{N}, j \geq 1$, we define iteratively two sequences of functions $u_{i, j}^{k, h}$ and $v_{i, j}^{k, h}$ as

$$
\begin{aligned}
u_{i, j}^{k, h} & :=\arg \min \left\{\mathcal{J}_{h}\left(u, v_{i, j-1}^{k, h}\right): u \in \mathcal{A}_{h}\left(w_{h}\left(t_{i}^{k}\right)\right)\right\}, \\
v_{i, j}^{k, h} & :=\arg \min \left\{\mathcal{J}_{h}\left(u_{i, j}^{k, h}, v\right): v \in \mathcal{F}_{h}, v \leq v_{i, j-1}^{k, h}\right\} .
\end{aligned}
$$

We notice that, since by assumption $v_{0}^{k, h} \geq 0$, combining Propositions 3.1 and 3.4 we deduce that (4.1) and (4.2) always admit unique solutions and, for every $k \in \mathbb{N}$ and every 
$i \in\{1, \ldots, k\}$, there exist $u_{i}^{k, h}, v_{i}^{k, h} \in \mathcal{F}_{h}$ such that $u_{i, j}^{k, h} \rightarrow u_{i}^{k, h}$ and $v_{i, j}^{k, h} \rightarrow v_{i}^{k, h}$ in $\mathcal{F}_{h}$ as $j \rightarrow+\infty$. Moreover, $0 \leq v_{i}^{k, h} \leq v_{i, j}^{k, h} \leq v_{i-1}^{k, h} \leq 1$ in $\Omega$ and, again thanks to Proposition 3.4,

$$
\begin{array}{ll}
\mathcal{J}_{h}\left(u_{i}^{k, h}, v_{i}^{k, h}\right) \leq \mathcal{J}_{h}\left(u, v_{i}^{k, h}\right) & \text { for every } u \in \mathcal{A}_{h}\left(w_{h}\left(t_{i}^{k}\right)\right), \\
\mathcal{J}_{h}\left(u_{i}^{k, h}, v_{i}^{k, h}\right) \leq \mathcal{J}_{h}\left(u_{i}^{k, h}, v\right) & \text { for every } v \in \mathcal{F}_{h}, v \leq v_{i}^{k} \text { in } \Omega .
\end{array}
$$

In the following proposition, we prove a finite-length property of the sequences $\left\{u_{i, j}^{k, h}\right\}$ and $\left\{v_{i, j}^{k, h}\right\}$ for $j \in \mathbb{N}$ and $i \in\{1, \ldots, k\}$.

Proposition 4.1 There exists $C_{h} \in(0,+\infty)$ such that for every $k \in \mathbb{N}$

$$
\sum_{i=1}^{k} \sum_{j=1}^{\infty}\left\|u_{i, j}^{k, h}-u_{i, j-1}^{k, h}\right\|_{H^{1}}+\left\|v_{i, j}^{k, h}-v_{i, j-1}^{k, h}\right\|_{H^{1}} \leq C_{h} .
$$

Proof In this proof, $C$ denotes a generic positive constant, which could change from line to line.

In view of (4.1)-(4.2) and of Proposition 3.5, we have that, for every $i \in\{1, \ldots, k\}$ and every $j \geq 2$

$$
\left\|u_{i, j}^{k, h}-u_{i, j-1}^{k, h}\right\|_{H^{1}} \leq C\left\|v_{i, j-1}^{k, h}-v_{i, j-2}^{k, h}\right\|_{\mathcal{F}_{h}} \leq C\left\|v_{i, j-1}^{k, h}-v_{i, j-2}^{k, h}\right\|_{1},
$$

where the second inequality is due to the equivalence of norms in finite dimension. For $j=1$, instead, by definition of $u_{j, 0}^{k, h}$ and of $v_{i, 0}^{k, h}$, we get

$$
\left\|u_{i, 1}^{k, h}-u_{i, 0}^{k, h}\right\|_{H^{1}} \leq C\left\|w_{h}\left(t_{i}^{k}\right)-w_{h}\left(t_{i-1}^{k}\right)\right\|_{H^{1}} .
$$

By definition (4.2) of $v_{i, j}^{k, h}$, we have that

$$
\sum_{j=1}^{\infty}\left\|v_{i, j}^{k, h}-v_{i, j-1}^{k, h}\right\|_{1}=\sum_{j=1}^{\infty} \int_{\Omega}\left(v_{i, j-1}^{k, h}-v_{i, j}^{k, h}\right) \mathrm{d} x=\int_{\Omega}\left(v_{i-1}^{k, h}-v_{i}^{k, h}\right) \mathrm{d} x .
$$

Thus, collecting inequalities (4.6)-(4.8), we obtain

$$
\begin{aligned}
& \sum_{i=1}^{k} \sum_{j=1}^{\infty}\left\|u_{i, j}^{k, h}-u_{i, j-1}^{k, h}\right\|_{H^{1}} \leq C \sum_{i=1}^{k} \int_{\Omega}\left(v_{i-1}^{k, h}-v_{i}^{k, h}\right) \mathrm{d} x+C \sum_{i=1}^{k}\left\|w_{h}\left(t_{i}^{k}\right)-w_{h}\left(t_{i-1}^{k}\right)\right\|_{H^{1}} \\
& \quad \leq C \int_{\Omega}\left(v_{0}^{k, h}-v_{k}^{k, h}\right) \mathrm{d} x+C \int_{0}^{T}\left\|\dot{w}_{h}(t)\right\|_{H^{1}} \mathrm{~d} t \leq C|\Omega|+C \int_{0}^{T}\left\|\dot{w}_{h}(t)\right\|_{H^{1}} \mathrm{~d} t .
\end{aligned}
$$

By equivalence of the norms in finite dimension, we obtain a similar inequality also in terms of phase-field $v$. Hence, being $w_{h} \in W^{1,2}\left([0, T] ; \mathcal{F}_{h}\right),(4.5)$ follows.

Following the lines of [32], we now construct a suitable arc-length parametrization of time and the functions $u_{k}^{h}, v_{k}^{h}$ interpolating between the values $u_{i-1}^{k, h}, u_{i}^{k, h}$ and $v_{i-1}^{k, h}, v_{i}^{k, h}$, respectively.

Let $s_{0}^{k}:=0$. For $i \geq 1$ and $j \in \mathbb{N}$, let

$$
\begin{aligned}
s_{i,-1}^{k} & :=s_{i-1}^{k}, \quad s_{i, 0}^{k}:=s_{i-1}^{k}+\tau_{k}, \\
s_{i, j+1 / 2}^{k} & :=s_{i, j}^{k}+\left\|u_{i, j+1}^{k, h}-u_{i, j}^{k, h}\right\|_{h, v_{i, j}^{k, h}}, \quad s_{i, j+1}^{k}:=s_{i, j+1 / 2}^{k}+\left\|v_{i, j+1}^{k, h}-v_{i, j}^{k, h}\right\|_{h, u_{i, j+1}^{k, h}} .
\end{aligned}
$$

We define $s_{i}^{k}:=\lim _{j} s_{i, j}^{k}$, which exists finite in view of Proposition 4.1 and Lemma A.1. 
We now define the interpolating functions $t_{k}^{h}, u_{k}^{h}$, and $v_{k}^{h}$, distinguishing between the three intervals $\left[s_{i,-1}^{k}, s_{i, 0}^{k}\right],\left[s_{i, j}^{k}, s_{i, j+1 / 2}^{k}\right]$, and $\left[s_{i, j+1 / 2}^{k}, s_{i, j+1}^{k}\right]$. For $s \in\left[s_{i,-1}^{k}, s_{i, 0}^{k}\right]$, we set

$$
\begin{aligned}
t_{k}^{h}(s) & :=t_{i-1}^{k}+s-s_{i,-1}^{k}, \\
u_{k}^{h}(s) & :=u_{i-1}^{k, h}+w_{h}\left(t_{k}^{h}(s)\right)-w_{h}\left(t_{i-1}^{k}\right), \\
v_{k}^{h}(s) & :=v_{i-1}^{k} .
\end{aligned}
$$

For $s \in\left[s_{i, j}^{k}, s_{i, j+1 / 2}^{k}\right]$ we define

$$
\begin{aligned}
& t_{k}^{h}(s):=t_{i}^{k}, \\
& u_{k}^{h}(s):=\left\{\begin{array}{l}
u_{i, j}^{k, h}+\left(s-s_{i, j}^{k}\right) \frac{u_{i, j+1}^{k, h}-u_{i, j}^{k, h}}{s_{i, j+1 / 2}^{k}-s_{i, j}^{k}} \text { if } s_{i, j+1 / 2}^{k} \neq s_{i, j}^{k}, \\
u_{i, j}^{k, h}=u_{i, j+1}^{k, h}
\end{array} \text { if } s_{i, j+1 / 2}^{k}=s_{i, j}^{k},\right. \\
& v_{k}^{h}(s):=v_{i, j}^{k, h} .
\end{aligned}
$$

Finally, for $s \in\left[s_{i, j+1 / 2}^{k}, s_{i, j+1}^{k}\right]$ we set

$$
\begin{aligned}
t_{k}^{h}(s) & :=t_{i}^{k}, \\
u_{k}^{h}(s) & :=u_{i, j+1}^{k, h}, \\
v_{k}^{h}(s) & :=\left\{\begin{array}{l}
v_{i, j}^{k, h}+\left(s-s_{i, j+1 / 2}^{k}\right) \frac{v_{i, j+1}^{k, h}-v_{i, j}^{k, h}}{s_{i, j+1}^{k}-s_{i, j+1 / 2}^{k}} \text { if } s_{i, j+1}^{k} \neq s_{i, j+1 / 2}^{k}, \\
v_{i, j}^{k}=v_{i, j+1}^{k}
\end{array} \text { if } s_{i, j+1}^{k}=s_{i, j+1 / 2}^{k} .\right.
\end{aligned}
$$

In this way, we have constructed a sequence of interpolating functions $\left(t_{k}^{h}, u_{k}^{h}, v_{k}^{h}\right):\left[0, S_{k}^{h}\right] \rightarrow$ $[0, T] \times \mathcal{F}_{h} \times \mathcal{F}_{h}$, where $S_{k}^{h}$ is the maximal value of the arc-length parameter $s$. By Proposition 4.1 and Lemma A.1, $S_{k}^{h}$ is uniformly bounded w.r.t. $k$. Moreover, we notice that

$$
t_{k}^{h}\left(s_{i}^{k}\right)=t_{i}^{k}, \quad u_{k}^{h}\left(s_{i}^{k}\right)=u_{i}^{k, h}, \quad v_{k}^{h}\left(s_{i}^{k}\right)=v_{i}^{k, h},
$$

and that there exists a positive constant $C$ independent of $k$ such that

$$
\left\|t_{k}^{h}\right\|_{W^{1, \infty}\left(\left[0, S_{k}^{h}\right] ;[0, T]\right)}+\left\|u_{k}^{h}\right\|_{W^{1,2}\left(\left[0, S_{k}^{h}\right] ; \mathcal{F}_{h}\right)}+\left\|v_{k}^{h}\right\|_{W^{1, \infty}\left(\left[0, S_{k}^{h}\right] ; \mathcal{F}_{h}\right)} \leq C .
$$

In the next proposition, we collect the stability properties and the energy balance satisfied by the interpolation functions $t_{k}^{h}, u_{k}^{h}$, and $v_{k}^{h}$.

Proposition 4.2 For every $k \in \mathbb{N}$ and every $i \in\{1, \ldots, k\}$ it holds

$$
\begin{array}{ll}
\mathcal{J}_{h}\left(u_{k}^{h}\left(s_{i}^{k}\right), v_{k}^{h}\left(s_{i}^{k}\right)\right) \leq \mathcal{J}_{h}\left(u, v_{k}^{h}\left(s_{i}^{k}\right)\right) & \text { for every } u \in \mathcal{A}_{h}\left(w_{h}\left(t_{h}^{k}\left(s_{i}^{k}\right)\right)\right), \\
\mathcal{J}_{h}\left(u_{k}^{h}\left(s_{i}^{k}\right), v_{k}^{h}\left(s_{i}^{k}\right)\right) \leq \mathcal{J}_{h}\left(u_{k}^{h}\left(s_{i}^{k}\right), v\right) & \text { for every } v \in \mathcal{F}_{h}, v \leq v_{k}^{h}\left(s_{i}^{k}\right) .
\end{array}
$$

Moreover, for every $s \in\left[0, S_{k}^{h}\right]$, we have

$$
\begin{aligned}
\mathcal{J}_{h}\left(u_{k}^{h}(s), v_{k}^{h}(s)\right)= & \mathcal{J}_{h}\left(u_{0, h}, v_{0, h}\right)-\int_{0}^{s}\left|\partial_{u} \mathcal{J}_{h}\right|\left(u_{k}^{h}(\sigma), v_{k}^{h}(\sigma)\right)\left\|\left(u_{k}^{h}\right)^{\prime}(\sigma)\right\|_{h, v_{k}^{h}(\sigma)} \mathrm{d} \sigma \\
& -\int_{0}^{s}\left|\partial_{u} \mathcal{J}_{h}\right|\left(u_{k}^{h}(\sigma), v_{k}^{h}(\sigma)\right)\left\|\left(v_{k}^{h}\right)^{\prime}(\sigma)\right\|_{h, u_{k}^{h}(\sigma)} \mathrm{d} \sigma \\
& +\int_{0}^{s} \int_{\Omega}\left(\mathrm{P}_{h}\left(v_{k}^{h}(\sigma)^{2}\right)+\eta\right) \nabla u_{k}^{h}(\sigma) \cdot \nabla \dot{w}\left(t_{k}^{h}(\sigma)\right)\left(t_{k}^{h}\right)^{\prime}(\sigma) \mathrm{d} x \mathrm{~d} \sigma .
\end{aligned}
$$


Proof In view of (4.12), the equilibrium conditions (4.14)-(4.15) are equivalent to (4.3) and (4.4).

As for (4.16), we need to show the energy balance in each interval of the form $\left[s_{i-1}^{k}, s_{i, 0}^{k}\right]$, $\left[s_{i, j}^{k}, s_{i, j+1 / 2}^{k}\right]$, and $\left[s_{i, j+1 / 2}^{k}, s_{i, j+1}^{k}\right]$. For every $\bar{s} \in\left[s_{i-1}^{k}, s_{i, 0}^{k}\right]$ we have, by chain-rule,

$$
\begin{aligned}
\mathcal{J}_{h}\left(u_{k}^{h}(\bar{s}), v_{k}^{h}(\bar{s})\right)= & \mathcal{J}_{h}\left(u_{k}^{h}\left(s_{i-1}^{k}\right), v_{k}^{h}\left(s_{i-1}^{k}\right)\right) \\
& +\int_{s_{i-1}^{k}}^{s_{i, 0}^{k}} \int_{\Omega}\left(\mathrm{P}_{h}\left(v_{k}^{h}(\sigma)^{2}\right)+\eta\right) \nabla u_{k}^{h}(\sigma) \cdot \nabla \dot{w}_{h}\left(t_{k}^{h}(\sigma)\right)\left(t_{k}^{h}\right)^{\prime}(\sigma) \mathrm{d} x \mathrm{~d} \sigma .
\end{aligned}
$$

For $\bar{s} \in\left[s_{i, j}^{k}, s_{i, j+1 / 2}^{k}\right]$, we recall the discrete energy balance (3.3) proved in Lemma 3.2, to which we apply the change of variable $r=\frac{\sigma-s_{i, j}^{k}}{s_{i, j+1 / 2}^{k}-s_{i, j}^{k}}$. Being $t_{k}^{h}$ and $v_{k}^{h}$ constant on the whole interval $\left[s_{i, j}^{k}, s_{i, j+1 / 2}^{k}\right]$, we get

$$
\begin{aligned}
\mathcal{J}_{h}\left(u_{k}^{h}(\bar{s}), v_{k}^{h}(\bar{s})\right)= & \mathcal{J}_{h}\left(u_{k}^{h}\left(s_{i, j}^{k}\right), v_{k}^{h}\left(s_{i, j}^{k}\right)\right) \\
& -\int_{s_{i, j}^{k}}^{s_{i, j+1 / 2}^{k}}\left|\partial_{u} \mathcal{J}_{h}\right|\left(u_{k}^{h}(\sigma), v_{k}^{h}(\sigma)\right)\left\|\left(u_{k}^{h}\right)^{\prime}(\sigma)\right\|_{h, v_{k}^{h}(\sigma)} \mathrm{d} \sigma .
\end{aligned}
$$

In a similar way, we can show that for every $\bar{s} \in\left[s_{i, j+1 / 2}^{k}, s_{i, j+1}^{k}\right]$

$$
\begin{aligned}
\mathcal{J}_{h}\left(u_{k}^{h}(\bar{s}), v_{k}^{h}(\bar{s})\right)= & \mathcal{J}_{h}\left(u_{k}^{h}\left(s_{i, j+1 / 2}^{k}\right), v_{k}^{h}\left(s_{i, j+1 / 2}^{k}\right)\right) \\
& -\int_{s_{i, j+1 / 2}^{k}}^{s_{i, j+1}^{k}}\left|\partial_{v} \mathcal{J}_{h}\right|\left(u_{k}^{h}(\sigma), v_{k}^{h}(\sigma)\right)\left\|\left(v_{k}^{h}\right)^{\prime}(\sigma)\right\|_{h, u_{k}^{h}(\sigma)} \mathrm{d} \sigma .
\end{aligned}
$$

Iterating equalities (4.17)-(4.19) we obtain (4.16).

We are now in a position to conclude the proof of Theorem 2.9.

Proof of Theorem 2.9 In view of inequality (4.13), we have that there exist $S_{h} \in(0,+\infty)$, $t_{h} \in W^{1, \infty}\left(\left[0, S_{h}\right] ;[0, T]\right), u_{h} \in W^{1,2}\left(\left[0, S_{h}\right] ; \mathcal{F}_{h}\right)$, and $v_{h} \in W^{1, \infty}\left(\left[0, S_{h}\right] ; \mathcal{F}_{h}\right)$ such that, up to a subsequence, $S_{k}^{h} \rightarrow S_{h}, t_{k}^{h} \rightarrow t_{h}$ weakly* in $W^{1, \infty}\left(\left[0, S_{h}\right] ;[0, T]\right), u_{k}^{h} \rightarrow u_{h}$ weakly in $W^{1,2}\left(\left[0, S_{h}\right] ; \mathcal{F}_{h}\right)$, and $v_{k}^{h} \rightarrow v_{h}$ weakly* in $W^{1, \infty}\left(\left[0, S_{h}\right] ; \mathcal{F}_{h}\right)$. In particular, we have that for every $s \in\left[0, S_{h}\right]$ and every sequence $s_{k}^{h} \rightarrow s$ it holds

$$
t_{k}^{h}\left(s_{k}^{h}\right) \rightarrow t_{h}(s), \quad u_{k}^{h}\left(s_{k}^{h}\right) \rightarrow u_{h}(s) \text { in } \mathcal{F}_{h}, \quad v_{k}^{h}\left(s_{k}^{h}\right) \rightarrow v_{h}(s) \text { in } \mathcal{F}_{h} .
$$

Moreover, $u_{h}^{\prime}(\cdot)=\dot{w}_{h}\left(t_{h}(\cdot)\right) t_{h}^{\prime}(\cdot)$ in $L^{2}\left(\left[0, S_{h}\right] ; \mathcal{F}_{h}\right)$.

Let us prove the stability conditions (2.31)-(2.32). Let $s \in\left(0, S_{h}\right)$ be such that $t_{h}^{\prime}(s)>0$. This implies that there exists a sequence of indices $i_{k} \in\{1, \ldots, k\}$ such that $s_{i_{k}}^{k} \rightarrow s$. Since the pair $\left(u_{k}^{h}\left(s_{i_{k}}^{k}\right), v_{k}^{h}\left(s_{i_{k}}^{k}\right)\right)$ satisfies (4.14)-(4.15) for every $k$, Lemma 3.3 implies (2.31)-(2.32). For $s=S_{h}$, the stability follows in the same way from the stability in $S_{k}^{h}$.

Finally, we have to show the energy equality. In order to do this, we pass to the limit in equality (4.16). Fix $s \in\left[0, S_{h}\right]$. In view of the convergences discussed above, we have that

$$
\begin{gathered}
\lim _{k} \int_{0}^{s} \int_{\Omega}\left(\mathrm{P}_{h}\left(v_{k}^{h}(\sigma)^{2}\right)+\eta\right) \nabla u_{k}^{h}(\sigma) \cdot \nabla \dot{w}\left(t_{k}^{h}(\sigma)\right)\left(t_{k}^{h}\right)^{\prime}(\sigma) \mathrm{d} x \mathrm{~d} \sigma \\
=\int_{0}^{s} \int_{\Omega}\left(\mathrm{P}_{h}\left(v_{h}(\sigma)^{2}\right)+\eta\right) \nabla u_{h}(\sigma) \cdot \nabla \dot{w}\left(t_{h}(\sigma)\right) t_{h}^{\prime}(\sigma) \mathrm{d} x \mathrm{~d} \sigma .
\end{gathered}
$$


As for the other two terms in the right-hand side of (4.16), we apply Lemma A.2 and [8, Theorem 3.1], which guarantees that

$$
\begin{aligned}
& \int_{0}^{s}\left|\partial_{u} \mathcal{J}_{h}\right|\left(u_{h}(\sigma), v_{h}(\sigma)\right)\left\|u_{h}^{\prime}(\sigma)\right\|_{h, v_{h}(\sigma)} \mathrm{d} \sigma \\
& \quad \leq \liminf _{k} \int_{0}^{s}\left|\partial_{u} \mathcal{J}_{h}\right|\left(u_{k}^{h}(\sigma), v_{k}^{h}(\sigma)\right)\left\|\left(u_{k}^{h}\right)^{\prime}(\sigma)\right\|_{h, v_{k}^{h}(\sigma)} \mathrm{d} \sigma, \\
& \quad \int_{0}^{s}\left|\partial_{v} \mathcal{J}_{h}\right|\left(u_{h}(\sigma), v_{h}(\sigma)\right)\left\|v_{h}^{\prime}(\sigma)\right\|_{h, u_{h}(\sigma)} \mathrm{d} \sigma \\
& \leq \liminf _{k} \int_{0}^{s}\left|\partial_{v} \mathcal{J}_{h}\right|\left(u_{k}^{h}(\sigma), v_{k}^{h}(\sigma)\right)\left\|\left(v_{k}^{h}\right)^{\prime}(\sigma)\right\|_{h, u_{k}^{h}(\sigma)} \mathrm{d} \sigma .
\end{aligned}
$$

Combining the previous inequalities and passing to the limsup in (4.16) as $k \rightarrow+\infty$ we deduce that

$$
\begin{aligned}
\mathcal{J}_{h}\left(u_{h}(s), v_{h}(s)\right) \leq & \mathcal{J}_{h}\left(u_{0, h}, v_{0, h}\right)-\int_{0}^{s}\left|\partial_{u} \mathcal{J}_{h}\right|\left(u_{h}(\sigma), v_{h}(\sigma)\right)\left\|u_{h}^{\prime}(\sigma)\right\|_{h, v_{h}(\sigma)} \mathrm{d} \sigma \\
& -\int_{0}^{s}\left|\partial_{v} \mathcal{J}_{h}\right|\left(u_{h}(\sigma), v_{h}(\sigma)\right)\left\|v_{h}^{\prime}(\sigma)\right\|_{h, u_{h}(\sigma)} \mathrm{d} \sigma \\
& +\int_{0}^{s} \int_{\Omega}\left(\mathrm{P}_{h}\left(v_{h}(\sigma)^{2}\right)+\eta\right) \nabla u_{h}(\sigma) \cdot \nabla \dot{w}\left(t_{h}(\sigma)\right) t_{h}^{\prime}(\sigma) \mathrm{d} x \mathrm{~d} \sigma
\end{aligned}
$$

In order to show the opposite inequality, we first apply the chain-rule to the energy function $\sigma \mapsto \mathcal{J}_{h}\left(u_{h}(\sigma), v_{h}(\sigma)\right)$, obtaining

$$
\begin{aligned}
\mathcal{J}_{h}\left(u_{h}(s), v_{h}(s)\right)= & \mathcal{J}_{h}\left(u_{0, h}, v_{0, h}\right)+\int_{0}^{s} \partial_{u} \mathcal{J}_{h}\left(u_{h}(\sigma), v_{h}(\sigma)\right)\left[u_{h}^{\prime}(\sigma)\right] \mathrm{d} \sigma \\
& +\int_{0}^{s} \partial_{v} \mathcal{J}_{h}\left(u_{h}(\sigma), v_{h}(\sigma)\right)\left[v_{h}^{\prime}(\sigma)\right] \mathrm{d} \sigma
\end{aligned}
$$

Being $\sigma \mapsto v_{h}(\sigma)$ non-increasing, we have that $\dot{v}_{h}(\sigma) \leq 0$ in $\Omega$. Hence, by definition of the slope (2.30) w.r.t. the phase-field $v$, we estimate the last term in the right-hand side of (4.22) with

$$
\int_{0}^{s} \partial_{v} \mathcal{J}_{h}\left(u_{h}(\sigma), v_{h}(\sigma)\right)\left[v_{h}^{\prime}(\sigma)\right] \mathrm{d} \sigma \geq-\int_{0}^{s}\left|\partial_{v} \mathcal{J}_{h}\right|\left(u_{h}(\sigma), v_{h}(\sigma)\right)\left\|v_{h}^{\prime}(\sigma)\right\|_{h, u_{h}(\sigma)} \mathrm{d} \sigma .
$$

As for the first integral term in (4.22), we rewrite it as

$$
\begin{aligned}
\int_{0}^{s} & \partial_{u} \mathcal{J}_{h}\left(u_{h}(\sigma), v_{h}(\sigma)\right)\left[u_{h}^{\prime}(\sigma)\right] \mathrm{d} \sigma \\
= & \int_{0}^{s} \partial_{u} \mathcal{J}_{h}\left(u_{h}(\sigma), v_{h}(\sigma)\right)\left[u_{h}^{\prime}(\sigma)-\dot{w}_{h}\left(t_{h}(\sigma)\right) t_{h}^{\prime}(\sigma)+\dot{w}_{h}\left(t_{h}(\sigma)\right) t_{h}^{\prime}(\sigma)\right] \mathrm{d} \sigma \\
= & \int_{0}^{s} \partial_{u} \mathcal{J}_{h}\left(u_{h}(\sigma), v_{h}(\sigma)\right)\left[u_{h}^{\prime}(\sigma)-\dot{w}_{h}\left(t_{h}(\sigma)\right) t_{h}^{\prime}(\sigma)\right] \mathrm{d} \sigma \\
& +\int_{0}^{s} \int_{\Omega}\left(\mathrm{P}_{h}\left(v_{h}(\sigma)^{2}\right)+\eta\right) \nabla u_{h}(\sigma) \cdot \nabla \dot{w}\left(t_{h}(\sigma)\right) t_{h}^{\prime}(\sigma) \mathrm{d} x \mathrm{~d} \sigma,
\end{aligned}
$$


and we notice that $u_{h}^{\prime}(\sigma)-\dot{w}_{h}\left(t_{h}(\sigma)\right) t_{h}^{\prime}(\sigma)=0$ on $\partial \Omega$ for $\sigma \in\left[0, S_{h}\right]$. In particular, whenever $t_{h}^{\prime}(\sigma)>0,(4.14)$ holds, so that

$$
\begin{array}{r}
\partial_{u} \mathcal{J}_{h}\left(u_{h}(\sigma), v_{h}(\sigma)\right)\left[u_{h}^{\prime}(\sigma)-\dot{w}_{h}\left(t_{h}(\sigma)\right) t_{h}^{\prime}(\sigma)\right] \\
=\left|\partial_{u} \mathcal{J}_{h}\right|\left(u_{h}(\sigma), v_{h}(\sigma)\right)\left\|u_{h}^{\prime}(\sigma)\right\|_{h, v_{h}(\sigma)}=0,
\end{array}
$$

where the second equality is due to the definition (2.29) of the slope w.r.t. the displacement $u$. On the other hand, when $t_{h}^{\prime}(\sigma)=0$, by (2.29) we have

$$
\begin{aligned}
& \partial_{u} \mathcal{J}_{h}\left(u_{h}(\sigma), v_{h}(\sigma)\right)\left[u_{h}^{\prime}(\sigma)-\dot{w}_{h}\left(t_{h}(\sigma)\right) t_{h}^{\prime}(\sigma)\right] \\
& \quad \geq-\left|\partial_{u} \mathcal{J}_{h}\right|\left(u_{h}(\sigma), v_{h}(\sigma)\right)\left\|u_{h}^{\prime}(\sigma)-\dot{w}_{h}\left(t_{h}(\sigma)\right) t_{h}^{\prime}(\sigma)\right\|_{h, v_{h}(\sigma)} \\
& \quad=-\left|\partial_{u} \mathcal{J}_{h}\right|\left(u_{h}(\sigma), v_{h}(\sigma)\right)\left\|u_{h}^{\prime}(\sigma)\right\|_{h, v_{h}(\sigma) .}
\end{aligned}
$$

Combining these two inequalities with (4.24), we deduce that

$$
\begin{aligned}
& \int_{0}^{s} \partial_{u} \mathcal{J}_{h}\left(u_{h}(\sigma), v_{h}(\sigma)\right)\left[u_{h}^{\prime}(\sigma)\right] \mathrm{d} \sigma \\
& \quad \geq-\int_{0}^{s}\left|\partial_{u} \mathcal{J}_{h}\right|\left(u_{h}(\sigma), v_{h}(\sigma)\right)\left\|u_{h}^{\prime}(\sigma)\right\|_{h, v_{h}(\sigma)} \mathrm{d} \sigma \\
& \quad+\int_{0}^{s} \int_{\Omega}\left(\mathrm{P}_{h}\left(v_{h}(\sigma)^{2}\right)+\eta\right) \nabla u_{h}(\sigma) \cdot \nabla \dot{w}\left(t_{h}(\sigma)\right) t_{h}^{\prime}(\sigma) \mathrm{d} x \mathrm{~d} \sigma .
\end{aligned}
$$

Finally, inserting (4.23) and (4.25) in (4.22), we get (2.33), and the proof is thus concluded.

\section{M-step algorithm}

We conclude this section discussing a variant of the above construction. Namely, we modify the infinite minimization algorithm (4.1)-(4.2) by stopping it after $M$ steps, with $M \in \mathbb{N}$ fixed a priori. Then, we define

$$
u_{i}^{k, h}:=u_{i, M}^{k, h} \quad \text { and } \quad v_{i}^{k, h}:=v_{i, M}^{k, h} .
$$

As in Proposition 4.1, we can show that there exists $C_{h} \in(0,+\infty)$ such that

$$
\sum_{i=1}^{k} \sum_{j=1}^{M}\left\|u_{i, j}^{k, h}-u_{i, j-1}^{k, h}\right\|_{H^{1}}+\left\|v_{i, j}^{k, h}-v_{i, j-1}^{k, h}\right\|_{H^{1}} \leq C_{h}
$$

uniformly w.r.t. $k$. This allows us to construct a time reparametrization as in (4.9)-(4.11) with $s_{0}^{k}:=0$ and, for $i \in\{1, \ldots, k\}$ and $j \in\{0, \ldots, M-1\}$,

$$
\begin{aligned}
s_{i,-1}^{k} & :=s_{i-1}^{k}, \quad s_{i, 0}^{k}:=s_{i-1}^{k}+\tau_{k}, \\
s_{i, j+1 / 2}^{k} & :=s_{i, j}^{k}+\left\|u_{i, j+1}^{k, h}-u_{i, j}^{k, h}\right\|_{h, v_{i, j}^{k, h}}, \quad s_{i, j+1}^{k, h}:=s_{i, j+1 / 2}^{k, h}+\left\|v_{i, j+1}^{k, h}-v_{i, j}^{k, h}\right\|_{h, u_{i, j+1}^{k, h}} .
\end{aligned}
$$

We set $s_{i}^{k}:=s_{i, M}^{k}$. In this way, we still obtain, for $h$ fixed, a sequence of triples $\left(t_{k}^{h}, u_{k}^{h}, v_{k}^{h}\right):\left[0, S_{k}^{h}\right] \rightarrow[0, T] \times \mathcal{F}_{h} \times \mathcal{F}_{h}$ satisfying the uniform bound (4.13) and the discrete energy balance (4.16). Moreover, thanks to (4.26), we have that $S_{k}^{h}$ is uniformly bounded. 
Up to a subsequence, we have that $S_{k}^{h} \rightarrow S_{h}, t_{k}^{h} \rightarrow t_{h}$ weakly* in $W^{1, \infty}\left(\left[0, S_{h}\right] ;[0, T]\right)$, $u_{k}^{h} \rightarrow u_{h}$ weakly in $W^{1,2}\left(\left[0, S_{h}\right] ; \mathcal{F}_{h}\right)$, and $v_{k}^{h} \rightarrow v_{h}$ weakly* in $W^{1, \infty}\left(\left[0, S_{h}\right] ; \mathcal{F}_{h}\right)$. As in the proof of Theorem 2.9, we can show that $\left(t_{h}, u_{h}, v_{h}\right)$ satisfies the energy balance (2.33).

In order to prove that $\left(t_{h}, u_{h}, v_{h}\right)$ is a parametrized finite-dimensional quasi-static evolution, we have to take care of the stability conditions (2.31)-(2.32), taking into account that this time the interpolation functions $t_{k}^{h}, u_{k}^{h}, v_{k}^{h}$ do not satisfy (4.14)-(4.15) at time $s_{i}^{k}=s_{i, M}^{k}$. However, given $s \in\left[0, S_{h}\right]$ such that $t_{h}^{\prime}(s)>0$, for every $k$ there exists an index $i_{k} \in\{1, \ldots, k\}$ such that $s \in\left[s_{i_{k}-1}^{k}, s_{i_{k}}^{k}\right]$. Up to subsequence, we have that $s_{i_{k}-1}^{k} \rightarrow \underline{s}$ and $s_{i_{k}}^{k} \rightarrow \bar{s}$ for some $0 \leq \underline{s} \leq \bar{s} \leq S_{h}$. Moreover, $s_{i_{k}, 0}^{k}=s_{i_{k}-1}^{k}+\tau_{k} \rightarrow \underline{s}$. We claim that $\underline{s}=\bar{s}$. Indeed, if by contradiction $\underline{s}<\bar{s}$, being $t_{k}^{h}$ constant in the interval $\left[s_{i_{k}, 0}^{k}, s_{i_{k}}^{k}\right]$, we get that $t_{h}$ is constant on $[\underline{s}, \bar{s}]$, which implies $t_{h}^{\prime}(s)=0$. Therefore, $s=\underline{s}=\bar{s}, s_{i_{k}, j}^{k} \rightarrow s$ for every $j \in\{-1, \ldots, M\}$, and the stability conditions (2.31)-(2.32) follows by Lemma 3.3.

Remark 4.3 We finally notice that the same construction can be applied to a slightly more general scheme, in which the number of steps in the alternate minimization algorithm is not fixed a priori for every $i \in\{1, \ldots, k\}$, but depends on the time node $t_{i}^{k}$. This fact is very important from a numerical point of view (see Sect. 6), since in general the algorithm (4.1)(4.2) has to be artificially stopped according to some criterion, which, at time $t_{i}^{k}$, is satisfied after a certain number $M_{i}^{k}$ of iterations. Thanks to the analysis described above, we are able to include all the possible stopping criteria.

\section{From space-discrete to space-continuous evolution}

This section is devoted to the proof of Theorem 2.4. In particular, we show that any limit of a sequence $\left(u_{h}, v_{h}\right):[0, T] \rightarrow \mathcal{F}_{h} \times \mathcal{F}_{h}$ of finite-dimensional quasi-static evolutions is a quasi-static evolution in the sense of Definition 2.3.

Before proving Theorem 2.4, we show two useful properties. Firstly, we state a uniform estimate on the operator $\mathrm{P}_{h}$. Secondly, we prove a stability property of the functionals $\mathcal{J}_{h}$ and $\mathcal{J}$ analogous to Lemma 3.3, and which takes into account the "convergence" of the finite-dimensional spaces $\mathcal{F}_{h}$ to $H^{1}(\Omega)$ as $h \rightarrow 0$.

Although the following result is standard, we provide its proof in "Appendix A" for the sake of completeness.

Lemma 5.1 Let $h>0$, let $\mathrm{P}_{h}: C(\bar{\Omega}) \rightarrow H^{1}(\Omega)$ be the operator defined by (2.19), and let $g \in C^{2}(\mathbb{R})$. Then, for every $M>0$ there exists a positive constant $C=C(g, M)$ depending only on $g$ and $M$ such that for every $v \in \mathcal{F}_{h}$ with $\|v\|_{\infty} \leq M$

$$
\left\|(g \circ v)-\mathrm{P}_{h}(g \circ v)\right\|_{1} \leq C h^{2}\|\nabla v\|_{2}^{2} .
$$

Proof See "Appendix A".

Lemma 5.2 For every $h>0$, let $w_{h}, u_{h}, v_{h} \in \mathcal{F}_{h}$ be such that $u_{h} \in \mathcal{A}_{h}\left(w_{h}\right), 0 \leq v_{h} \leq 1$ in $\Omega$, and

$$
\begin{array}{ll}
\mathcal{J}_{h}\left(u_{h}, v_{h}\right) \leq \mathcal{J}_{h}\left(u, v_{h}\right) & \text { for every } u \in \mathcal{A}_{h}\left(w_{h}\right), \\
\mathcal{J}_{h}\left(u_{h}, v_{h}\right) \leq \mathcal{J}_{h}\left(u_{h}, v\right) & \text { for every } v \in \mathcal{F}_{h} \text { such that } v \leq v_{h} .
\end{array}
$$


Assume that $w_{h} \rightarrow \bar{w}$ in $H^{1}(\Omega), u_{h} \rightarrow \bar{u}$ and $v_{h} \rightarrow \bar{v}$ weakly in $H^{1}(\Omega)$ as $h \rightarrow 0$. Then $\bar{u} \in \mathcal{A}(\bar{w})$ and

$$
\begin{array}{ll}
\mathcal{J}(\bar{u}, \bar{v}) \leq \mathcal{J}(u, \bar{v}) & \text { for every } u \in \mathcal{A}(\bar{w}), \\
\mathcal{J}(\bar{u}, \bar{v}) \leq \mathcal{J}(\bar{u}, v) & \text { for every } v \in H^{1}(\Omega) \text { with } v \leq \bar{v} .
\end{array}
$$

Moreover, $u_{h} \rightarrow \bar{u}$ strongly in $H^{1}(\Omega)$.

Proof Let us prove (5.4). Let $u \in H^{1}(\Omega)$ be such that $u=\bar{w}$ on $\partial \Omega$. Thanks to (2.14) and to the interpolation error estimates in, e.g., [38, Theorem 3.4.2], for every $\varphi \in C_{c}^{\infty}(\Omega)$ there exists a sequence $\varphi_{h} \in \mathcal{F}_{h}$ such that $\varphi_{h}=0$ on $\partial \Omega$ and $\varphi_{h} \rightarrow \varphi$ in $H^{1}(\Omega)$ as $h \rightarrow 0$. Let us consider as a competitor in (5.2) the function $\psi_{h}:=\varphi_{h}+w_{h}$. For such a $\psi_{h}$ we have

$$
\int_{\Omega}\left(\mathrm{P}_{h}\left(v_{h}^{2}\right)+\eta\right)\left|\nabla u_{h}\right|^{2} \mathrm{~d} x \leq \int_{\Omega}\left(\mathrm{P}_{h}\left(v_{h}^{2}\right)+\eta\right)\left|\nabla \psi_{h}\right|^{2} \mathrm{~d} x .
$$

It is clear that $\psi_{h} \rightarrow \psi:=\varphi+\bar{w}$ in $H^{1}(\Omega)$ and, by Lemma $5.1, \mathrm{P}_{h}\left(v_{h}^{2}\right) \rightarrow \bar{v}^{2}$ strongly in $L^{p}(\Omega)$ for every $p \in[1,+\infty)$. Therefore, applying [25, Theorem 7.5] and passing to the limit as $h \rightarrow 0$ in (5.6), we get

$$
\begin{aligned}
\int_{\Omega}\left(\bar{v}^{2}+\eta\right)|\nabla \bar{u}|^{2} \mathrm{~d} x & \leq \liminf _{h \rightarrow 0} \int_{\Omega}\left(\mathrm{P}_{h}\left(v_{h}^{2}\right)+\eta\right)\left|\nabla u_{h}\right|^{2} \mathrm{~d} x \\
& \leq \limsup _{h \rightarrow 0} \int_{\Omega}\left(\mathrm{P}_{h}\left(v_{h}^{2}\right)+\eta\right)\left|\nabla \psi_{h}\right|^{2} \mathrm{~d} x \\
& \leq \int_{\Omega}\left(\bar{v}^{2}+\eta\right)|\nabla \psi|^{2} \mathrm{~d} x .
\end{aligned}
$$

By density, we have that the chain of inequalities (5.7) holds for every $\psi \in \mathcal{A}(\bar{w})$. Moreover, it is easy to see that (5.7) is equivalent to (5.4).

Specifying (5.7) for $\psi=\bar{u}$, we get that

$$
\lim _{h \rightarrow 0} \int_{\Omega}\left(\mathrm{P}_{h}\left(v_{h}^{2}\right)+\eta\right)\left|\nabla u_{h}\right|^{2} \mathrm{~d} x=\int_{\Omega}\left(\bar{v}^{2}+\eta\right)|\nabla \bar{u}|^{2} \mathrm{~d} x
$$

which implies the strong convergence of $u_{h}$ to $\bar{u}$ in $H^{1}(\Omega)$.

We now prove (5.5). Let us first consider a competitor $v \in H^{1}(\Omega) \cap L^{\infty}(\Omega), v \leq \bar{v}$ in $\Omega$. Let $\varphi^{k} \in C^{\infty}(\bar{\Omega})$ be such that $\varphi^{k} \leq 0$ in $\Omega$ and $\varphi^{k} \rightarrow v-\bar{v}$ in $H^{1}(\Omega)$ as $k \rightarrow+\infty$. Let us set $v_{h}^{k}:=\mathrm{P}_{h}\left(\varphi^{k}\right)+v_{h}$. Then, $v_{h}^{k} \leq v_{h}$ for every $h>0$ and every $k \in \mathbb{N}, v_{h}^{k} \in \mathcal{F}_{h}$, and $v_{h}^{k} \rightarrow \bar{v}+\varphi^{k}$ weakly in $H^{1}(\Omega)$ as $h \rightarrow 0$. By the quadratic structure of $\mathcal{J}_{h}$, by (5.3), and by the definition of $v_{h}^{k}$,

$$
\begin{aligned}
& \frac{1}{2} \int_{\Omega}\left(\mathrm{P}_{h}\left(v_{h}^{2}\right)+\eta\right)\left|\nabla u_{h}\right|^{2} \mathrm{~d} x+\frac{1}{2} \int_{\Omega} \mathrm{P}_{h}\left(\left(1-v_{h}\right)^{2}\right) \mathrm{d} x \\
& \leq \frac{1}{2} \int_{\Omega}\left(\mathrm{P}_{h}\left(\left(v_{h}^{k}\right)^{2}\right)+\eta\right)\left|\nabla u_{h}\right|^{2} \mathrm{~d} x+\frac{1}{2} \int_{\Omega} \mathrm{P}_{h}\left(\left(1-v_{h}^{k}\right)^{2}\right) \mathrm{d} x \\
& \quad+\frac{1}{2} \int_{\Omega}\left|\nabla \mathrm{P}_{h}\left(\varphi^{k}\right)\right|^{2} \mathrm{~d} x+\int_{\Omega} \nabla \mathrm{P}_{h}\left(\varphi^{k}\right) \cdot \nabla v_{h} \mathrm{~d} x .
\end{aligned}
$$

Since $u_{h} \rightarrow \bar{u}$ and $\mathrm{P}_{h}\left(\varphi^{k}\right) \rightarrow \varphi^{k}$ strongly in $H^{1}(\Omega), \mathrm{P}_{h}\left(v_{h}^{2}\right) \rightarrow \bar{v}^{2}$ and $\mathrm{P}_{h}\left(\left(v_{h}^{k}\right)^{2}\right) \rightarrow$ $\left(\varphi^{k}+\bar{v}\right)^{2}$ strongly in $L^{p}(\Omega)$ for every $p \in[1,+\infty)$ (see Lemma 5.1), and $v_{h}-\bar{v}$ weakly in $H^{1}(\Omega)$, passing to the limit as $h \rightarrow 0$ in (5.8) we deduce that 


$$
\begin{aligned}
& \frac{1}{2} \int_{\Omega}\left(\bar{v}^{2}+\eta\right)|\nabla \bar{u}|^{2} \mathrm{~d} x+\frac{1}{2} \int_{\Omega}(1-\bar{v})^{2} \mathrm{~d} x \\
& \quad \leq \frac{1}{2} \int_{\Omega}\left(\left(\varphi^{k}+\bar{v}\right)^{2}+\eta\right)|\nabla \bar{u}|^{2} \mathrm{~d} x+\frac{1}{2} \int_{\Omega}\left(1-\left(\varphi^{k}+\bar{v}\right)\right)^{2} \mathrm{~d} x \\
& \quad+\frac{1}{2} \int_{\Omega}\left|\nabla \varphi^{k}\right|^{2} \mathrm{~d} x+\int_{\Omega} \nabla \varphi^{k} \cdot \nabla \bar{v} \mathrm{~d} x .
\end{aligned}
$$

If we let $k \rightarrow+\infty$ in (5.9), recalling that $\varphi^{k} \rightarrow v-\bar{v}$ in $H^{1}(\Omega)$, we obtain

$$
\begin{aligned}
& \frac{1}{2} \int_{\Omega}\left(\bar{v}^{2}+\eta\right)|\nabla \bar{u}|^{2} \mathrm{~d} x+\frac{1}{2} \int_{\Omega}(1-\bar{v})^{2} \mathrm{~d} x \\
& \leq \frac{1}{2} \int_{\Omega}\left(v^{2}+\eta\right)|\nabla \bar{u}|^{2} \mathrm{~d} x+\frac{1}{2} \int_{\Omega}(1-v)^{2} \mathrm{~d} x \\
& \quad+\frac{1}{2} \int_{\Omega}|\nabla(v-\bar{v})|^{2} \mathrm{~d} x+\int_{\Omega} \nabla(v-\bar{v}) \cdot \nabla \bar{v} \mathrm{~d} x .
\end{aligned}
$$

Rearranging the last two terms in the right-hand side of (5.10), we get the stability condition (5.5) for $v \in H^{1}(\Omega) \cap L^{\infty}(\Omega)$ with $v \leq \bar{v}$. By a truncation argument, we get the same conclusion for $v \in H^{1}(\Omega)$ with $v \leq \bar{v}$.

We are now ready to prove Theorem 2.4.

Proof of Theorem 2.4 As already mentioned in Remark 2.5, in order to prove the existence of a quasi-static evolution in the sense of Definition 2.3, we show that any sequence of finitedimensional quasi-static evolutions $\left(u_{h}, v_{h}\right):[0, T] \rightarrow \mathcal{F}_{h}$ converges, up to a subsequence, to a quasi-static evolution as the mesh parameter $h$ tends to 0 .

For every $h>0$, we need first to find the right sequence of finite-dimensional quasi-static evolutions $\left(u_{h}, v_{h}\right)$ starting from a suitable initial datum $u_{0, h}, v_{0, h} \in \mathcal{F}_{h}$ and with a suitable boundary Dirichlet condition $w_{h}:[0, T] \rightarrow \mathcal{F}_{h}$.

As mentioned in Sect. 2, there exists a sequence $w_{h} \in W^{1,2}\left([0, T] ; H^{1}(\Omega)\right)$ such that $w_{h} \in W^{1,2}\left([0, T] ; \mathcal{F}_{h}\right)$ and $w_{h} \rightarrow w$ in $W^{1,2}\left([0, T] ; H^{1}(\Omega)\right)$ as $h \rightarrow 0$ (see [38]). In particular, the last convergence implies that $w_{h}(t) \rightarrow w(t)$ in $H^{1}(\Omega)$ for every $t \in[0, T]$ and $\dot{w}_{h}(t) \rightarrow \dot{w}(t)$ in $H^{1}(\Omega)$ for a.e. $t \in[0, T]$. Again by [38], we can also find two sequences $u_{0, h}, v_{0, h} \in \mathcal{F}_{h}$ such that $u_{0, h} \in \mathcal{A}_{h}\left(w_{h}(0)\right), 0 \leq v_{0, h} \leq 1$ in $\Omega$, and, as $h \rightarrow 0$, $u_{0, h} \rightarrow u_{0}$ and $v_{0, h} \rightarrow v_{0}$ in $H^{1}(\Omega)$.

By Corollary 2.10, for every $h>0$ there exists a finite-dimensional quasi-static evolution $\left(u_{h}, v_{h}\right):[0, T] \rightarrow \mathcal{F}_{h} \times \mathcal{F}_{h}$ with $u_{h}(0)=u_{0, h}, v_{h}(0)=v_{0, h}$, and $u_{h}(t) \in \mathcal{A}\left(w_{h}(t)\right)$ for every $t \in[0, T]$.

In view of (2.24)-(2.26) and of the construction of $u_{0, h}, v_{0, h}$, and $w_{h}$, we have that

$$
\sup _{\substack{h>0 \\ t \in[0, T]}}\left\|u_{h}(t)\right\|_{H^{1}}<+\infty \quad \text { and } \quad \sup _{\substack{h>0 \\ t \in[0, T]}}\left\|v_{h}(t)\right\|_{H^{1}}<+\infty .
$$

Since the sequence $v_{h}:[0, T] \rightarrow H^{1}(\Omega)$ is such that (5.11) holds, $t \mapsto v_{h}(t)$ is nonincreasing, and, for every $t \in[0, T], v_{h}(t)$ takes values in $[0,1]$, applying a generalized version of Helly's Selection Theorem (see [24, Theorem 2.3], we find a non-increasing function $v:[0, T] \rightarrow H^{1}(\Omega)$ such that, along a suitable subsequence $h_{k} \rightarrow 0$, for every $t \in[0, T] v_{h_{k}}(t)$ converges to $v(t)$ weakly in $H^{1}(\Omega)$ and strongly in $L^{p}(\Omega)$ for every $p \in[1,+\infty)$. In particular, $v(0)=v_{0}$ and $0 \leq v(t) \leq 1$ in $\Omega$ for every $t \in[0, T]$, hence condition (1) of Definition 2.3 is satisfied. 
In view of (5.11), for every $t \in[0, T]$, we have that, up to a subsequence (possibly dependent on $t), u_{h_{k}}(t) \rightarrow u(t)$ weakly in $H^{1}(\Omega)$ for some $u(t) \in \mathcal{A}(w(t))$. By Lemma 5.2, we deduce that the pair $(u(t), v(t))$ satisfies the stability conditions (2.9) and (2.10), and $u_{h_{k}}(t) \rightarrow u(t)$ strongly in $H^{1}(\Omega)$. Moreover, by Lemma 5.2 and by uniqueness of solution of the minimum problem

$$
\min \left\{\mathcal{J}(u, v(t)): u \in H^{1}(\Omega), u \in \mathcal{A}(w(t))\right\},
$$

we have that the whole sequence $u_{h_{k}}(t)$ converges to $u(t)$ strongly in $H^{1}(\Omega)$ for every $t \in[0, T]$.

Finally, in order to prove the energy inequality (2.11), we need to pass to the limit in the finite-dimensional energy inequality (2.26) as $h_{k} \rightarrow 0$. Since $u_{0, h} \rightarrow u_{0}$ and $v_{0, h} \rightarrow v_{0}$ and Lemma 5.1 holds, we have that $\mathcal{J}_{h_{k}}\left(u_{0, h_{k}}, v_{0, h_{k}}\right) \rightarrow \mathcal{J}\left(u_{0}, v_{0}\right)$. Again by Lemma 5.1, $\mathrm{P}_{h_{k}}\left(v_{h_{k}}^{2}(t)\right) \rightarrow v(t)$ in $L^{p}(\Omega)$ for every $p \in[1,+\infty)$ and every $t \in[0, T]$. Finally, by construction, $w_{h} \rightarrow w$ in $W^{1,2}\left([0, T] ; H^{1}(\Omega)\right)$. Hence, passing to the limit in (2.26) as $h_{k} \rightarrow 0$ and applying the dominated convergence theorem, we get (2.11), and this concludes the proof of the theorem.

\section{Numerical experiments}

In this section, we illustrate numerically the previous findings simulating brittle fracture propagation. We remark that the purpose of this section is not to challenge the efficiency of the numerical methods, but rather to show the consistency of the previously discussed theory. We keep an extensive numerical analysis for future work.

For the following numerical experiments, we make use of the experiences gained from previous calculations in $[5,6,10,13,19]$, where we also find some of the examples used herein. Below we use the original Ambrosio-Tortorelli functional $\mathcal{J}_{\varepsilon}$ defined in (1.1), whose discretized version we still denote by $\mathcal{J}_{h}$. We recall it here for the reader's convenience: for all $u, v \in \mathcal{F}_{h}$

$$
\mathcal{J}_{h}(u, v):=\frac{1}{2} \int_{\Omega}\left(\mathrm{P}_{h}\left(v^{2}\right)+\eta_{\varepsilon}\right)|\nabla u|^{2} \mathrm{~d} x+\int_{\Omega}\left(\kappa \varepsilon|\nabla v|^{2}+\frac{\kappa}{4 \varepsilon} \mathrm{P}_{h}\left((1-v)^{2}\right)\right) \mathrm{d} x .
$$

The following implementations are made with Freefem ++ and are performed on a MacBook Pro, 2.6 GHz Intel Core i7, 8 GB 1600 MHz DDR3.

For all the examples in this section, we fix the basic domain $\Omega:=(0,1) \times(0,1)$ and we choose a fixed time step size $\tau$. Hence, with an initial time $t_{0}:=0$ we set $t_{i}:=i \tau$. By $v_{i}$ and $u_{i}$, we denote the phase and the displacement fields at time $t_{i}$, respectively. We present three simulations for different boundary data $w$ and initial cracks $\Gamma$, imposed by an initial phase-field of the form

$$
v_{0}(x):=1-\exp \left(-\frac{\operatorname{dist}(x, \Gamma)}{2 \varepsilon}\right) .
$$

The specific choice made in (6.2) comes from the fact that $v_{0}$ is a recovery sequence for the $\Gamma$-limit of $\mathcal{J}_{\varepsilon}$ (see, e.g., $[3,15]$ ). We stress once again the physical interpretation of the phase-field: Where it is close to zero the crack appears. On the contrary, where it takes the value one the elastic body is perfectly sound. As it follows from the proof of $\Gamma$-convergence of the functional $\mathcal{J}_{\varepsilon}$, the fracture theoretically gains a thickness of order $\varepsilon$. However, in our simulations, we plot only the phase-field variable without giving a precise description of the crack set. 
We notice that initializing the pre-crack by an initial phase-field is a crucial difference compared to the implementations in the existing literature, where the initial crack is imposed by a notch in the domain. Moreover, in the functional $\mathcal{J}_{h}$, we keep the projection operator $\mathrm{P}_{h}$, which is dropped in the cited works. However, with these techniques, we stay closer to the theoretical framework, which is more suitable for our purpose.

We repeat the basic algorithm that we want to implement from (4.1)-(4.2) dropping the indices $k$ and $h$ and adding a stopping criterion as described at the end of Sect. 4. Given $i \geq 1$ and the phase-field $v_{i-1}$ at time $t_{i-1}$, we set $v_{i, 0}:=v_{i-1}$, and we define inductively on $j \geq 1$

$$
\begin{aligned}
& u_{i, j}:=\arg \min \left\{\mathcal{J}_{h}\left(u, v_{i, j-1}\right): u \in \mathcal{A}_{h}\left(w_{h}\left(t_{i}\right)\right)\right\}, \\
& v_{i, j}:=\arg \min \left\{\mathcal{J}_{h}\left(u_{i, j}, v\right): v \in \mathcal{F}_{h}, v \leq v_{i, j-1}\right\} .
\end{aligned}
$$

Due to the convergence of both sequences as $j \rightarrow+\infty$, we stop the loop when the phasefield does not show any significant changes. More precisely, we fix a threshold $0<\operatorname{Tol}_{v} \ll$ 1 , and we perform the alternate minimization (6.3)-(6.4) until we find $\bar{j} \in \mathbb{N}$ such that $\left\|v_{i, \bar{j}}-v_{i, \bar{j}-1}\right\|_{\infty} \leq \operatorname{Tol}_{v}$ and $\operatorname{set}\left(u_{i}, v_{i}\right):=\left(u_{i, \bar{j}}, v_{i, \bar{j}}\right)$.

The minimum in (6.3) is simply obtained by solving the linear Euler-Lagrange equation. The numerical treatment of the irresistibility condition $v \leq v_{i, j-1}$ in (6.4), instead, is an issue to be discussed. In many papers (see, for instance, $[6,11,13,18]$ ), this constraint has been replaced by a Dirichlet boundary condition, forcing $v$ to be zero where $v_{i, j-1}$ is below a certain threshold. The method turned out to be numerically very efficient. However, up to our knowledge, there is a lack of a rigorous theoretical proof of convergence of the scheme in the framework of quasi-static evolution problems.

In order to be as close as possible to the theoretical results discussed in this paper, in our numerical simulations, we do not exploit the algorithm proposed in $[11,13]$, but we rather perform the constrained minimization by a projected Newton method as in [19]. We precisely use the algorithm presented in [31, Sect. 5.5.2] (see also [9]). Due to the quadratic structure of the functional $\mathcal{J}_{h}(u, \cdot)$ this procedure is not too expensive.

On most parts of the domain the phase-field will be nearly constant. Only close to the crack it is expected to be very steep. To get an appropriate interpolation error, the mesh has to be very fine in the neighborhood of the crack, while it can be coarse elsewhere. Thus, we use an adaptive triangulation refining the mesh where it is necessary. For our purposes, we regularly adapt the mesh in the iteration procedure exploiting the standard routine implemented in Freefem++, which uses an anisotropic interpolation error estimator.

We notice that in the theoretical part of this paper, the mesh $\mathcal{T}_{h}$ is not allowed to vary. Working with a constant mesh throughout the whole algorithm would require it to be extremely fine on the entire domain in order not to influence the propagation of the crack path. With an adaptive procedure, instead, we can omit this difficulty and we can save a lot of computational effort. Moreover, we mention that such an approach has been already investigated and validated in $[6,18]$.

Concerning the choice of the mesh, we have theoretically considered two other restrictions that we do not take into account in the numerical simulations. Firstly, we require (2.17). This condition is only needed to ensure that the phase-field is non-negative (see Proposition 3.1). Nevertheless, even not making any restriction on the mesh triangles, the non-negativity of the phase-field turns out to be a posteriori fulfilled. Secondly, in (2.14) a certain isotropy of the triangles is required in order to guarantee the usual a priori interpolation estimate. However, looking at the final mesh, we observe that the assertion is fulfilled simply because the mesh consists of finitely many triangles. 
Table 1 Numerical parameters

\begin{tabular}{llllll}
\hline$\tau$ & $\varepsilon$ & $\eta_{\varepsilon}$ & $\kappa$ & $T o l_{v}$ & Tol $_{\text {mesh }}$ \\
\hline 0.01 & $2 \cdot 10^{-3}$ & $10^{-5}$ & 0.5 & $2 \cdot 10^{-3}$ & $2 \cdot 10^{-3}$ \\
\hline
\end{tabular}

The basic numerical scheme that we use is described in Algorithm 1. As far as not explicitly differently stated, we use the numerical parameters as summarized in Table 1. As an output, at each time step, we visualize the number of alternating minimizations as well as the total number of steps of the projected Newton method necessary to compute the minimizers in line 9 of Algorithm 1. Moreover, we plot the crack length as a function of time, approximating it by $\frac{1}{4 \varepsilon} \int_{\Omega}\left(1-v_{i}\right)^{2} \mathrm{~d} x+\varepsilon \int_{\Omega}\left|\nabla v_{i}\right|^{2} \mathrm{~d} x$.

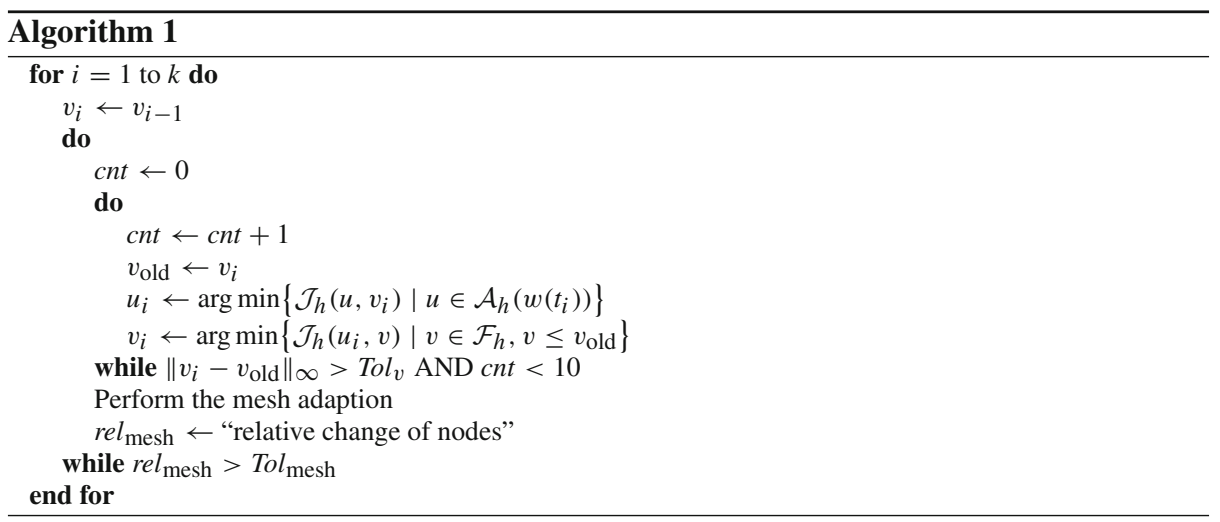

In Algorithm 1, we use a scheme similar to the one in [5]. We also ensure a mesh adaption after at most 10 alternations, which reduces the number of minimizations compared to a procedure where the mesh is adapted only after the solution on the current mesh has been computed. In our scheme, for each adaptation, the mesh is completely renewed in such a way that unnecessary nodes may be removed, reducing significantly the total number of nodes. The "relative change of nodes" $\mathrm{rel}_{\text {mesh }}$ in Algorithm 1 refers to the quotient of the number of added nodes and the number of nodes of the old mesh. In this way, the difference between two consecutive meshes is quantified and the alternating procedure continues until $\mathrm{rel}_{\text {mesh }}$ goes below a certain threshold $T o l_{\text {mesh }}$.

Remark 6.1 We point out that one could also couple the adaptation of the mesh closer to the single minimization step, as proposed in $[18,20]$. Therein, the mesh adaption is based on a residual estimate of the Fréchet derivative of the energy functional and allows only refinements of the mesh. In this way, the authors could prove - under certain additional assumptions - that their scheme converges to a critical point as $h \rightarrow 0$ (see [18, Theorem 4.1]).

\section{First example}

Our first example starts from a fully symmetrical setting, where we impose an initial crack orthogonal to and in the middle of the left boundary of the domain $\Omega$ described by the set

$$
\Gamma_{1}:=\left[0, \frac{1}{4}\right] \times\left\{\frac{1}{2}\right\} .
$$




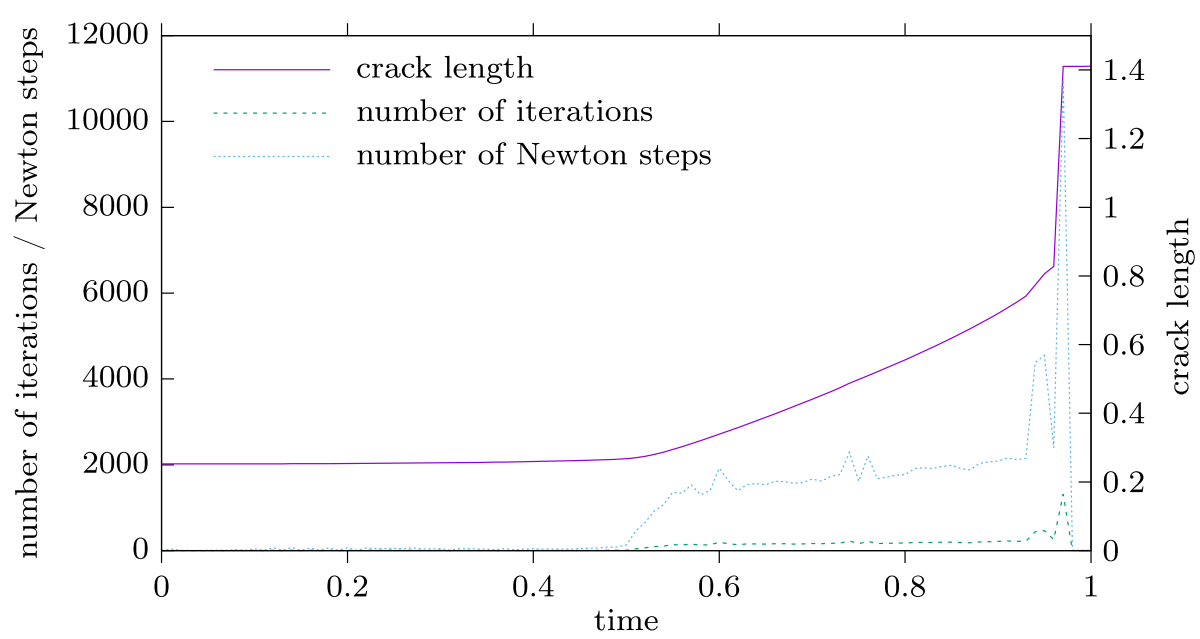

Fig. 1 Numerical output data of the first example with initial crack $\Gamma_{1}$ and boundary condition $w_{1}$

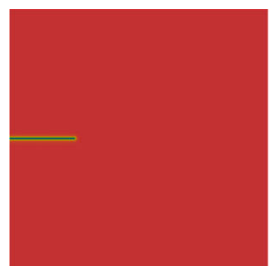

$t=0$

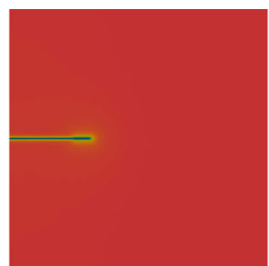

$t=0.6$

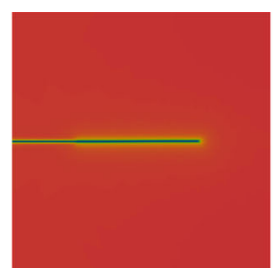

$t=0.96$

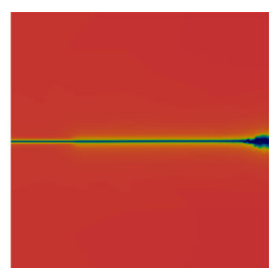

$t=0.97$

Fig. 2 Phase-field of first example at various times

The crack is initialized by choosing the phase-field $v_{0}$ in (6.2) for $\Gamma=\Gamma_{1}$. We also use the symmetrical boundary condition

$$
w_{1}(t)= \begin{cases}t & \text { on }\{0\} \times\left[0, \frac{1}{2}\right), \\ -t & \text { on }\{0\} \times\left(\frac{1}{2}, 1\right] .\end{cases}
$$

The measured values are visualized in Figure 1. The crack propagates until the domain is fully broken at time $t=0.97$. In Figs. 2 and 3, one can observe the corresponding phase-field at different time steps and the generated mesh, respectively.

For this simulation, there were in total 9874 alternations and 96,104 Newton steps computed, which means that 105,978 linear systems have been solved. The final mesh has 71,629 nodes.

The presented setting is quite standard, and our result seems consistent compared to the ones in the literature. Note that a quasi-static evolution does not need to be continuous. Indeed, the crack jumps instantaneously at the last time step. Due to the large change in the phase field, the algorithm needs many iterations to complete, as it can be noticed from the peaks at time $t=0.97$ in Fig. 1 .

One realizes from Fig. 1 that the phase-field spreads close to the right part of the boundary of $\Omega$. This effect can be observed in all the examples. The reason for this phenomenon is that the irreversibility condition is imposed by using the intermediate step $v_{i, j-1}$ as an upper bound, which does not refer to a real physical state. Using another upper bound, such 
Fig. 3 Mesh at time $t=0.6$ used for the first example

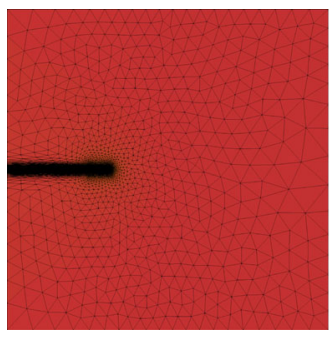

Complete mesh

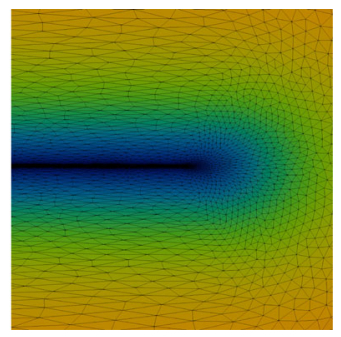

Mesh at the crack tip

as $v_{i-1}$, would eliminate this effect as it can be observed, e.g., in [6,7,18-20], but would break the consistency with our theory. Particularly, replacing $v_{i, j-1}$ by $v_{i-1}$ in the inequality constraint in (6.4) still let $\left(u_{i, j}, v_{i, j}\right)$ converge as $j \rightarrow+\infty$ (up to subsequence) to a critical point of $J_{h}(u, v)$ (see [19, Proposition 2]). However, we were not able to prove the energydissipation equality (2.33) for this kind of constraint.

\section{Second example}

In our second example, we break the symmetry by turning the initial crack into a set not orthogonal to the left boundary and shifted out of the middle point. Namely, we use the initial phase-field $v_{0}$ from (6.2) with $\Gamma$ replaced by

$$
\Gamma_{2}:=\left\{(x, y) \in \mathbb{R}^{2} \mid x \in\left[0, \frac{1}{4}\right], y=\frac{1}{2} x+\frac{1}{4}\right\} .
$$

The boundary condition stays the same in the sense that it is $t$ below the crack and $-t$ above it. Thus, we have

$$
w_{2}(t)= \begin{cases}t & \text { on }\{0\} \times\left[0, \frac{1}{4}\right), \\ -t & \text { on }\{0\} \times\left(\frac{1}{4}, 1\right] .\end{cases}
$$

With this settings, the specimen completely breaks at time $t=1.05$, where the crack crosses the bottom border (see Fig. 5). Figure 4 shows the corresponding numerical measurements. In this case, we can observe a number of peaks appearing before the final jump of the fracture. The phenomena might be explained, as in the previous example, by the fact that the phase-field is actually experiencing jumps of different size at each time step, as it can be noticed by the oscillations in the graph of the crack length.

For this example, we do not know any comparable test case in the literature to refer to. Since the crack tip is not in the middle of the domain and the boundary condition is not balanced, the shown crack path seems reasonable.

The total number of alternating minimizations until the crack completes is 10,222 , and 96,528 Newton steps were performed. Thus, the simulation solved 106,750 linear systems. The mesh at the time of crack completion is composed of 108,020 nodes (Fig. 6).

During our numerical analysis, we realized that one can find significantly different phase fields by varying $\varepsilon$ (see Fig. 7). The smaller $\varepsilon$ is, the less the crack path turns downwards. For the value $10^{-3}$, it even leaves the specimen at its right border. Since the phase-field endows the crack with a non-physical width of order $\varepsilon$, the crack set is influenced by the boundary of the domain for large values of this parameter. The effect is generally not unexpected and has already been observed in $[6,18]$, where the authors performed a parameter sensitivity 


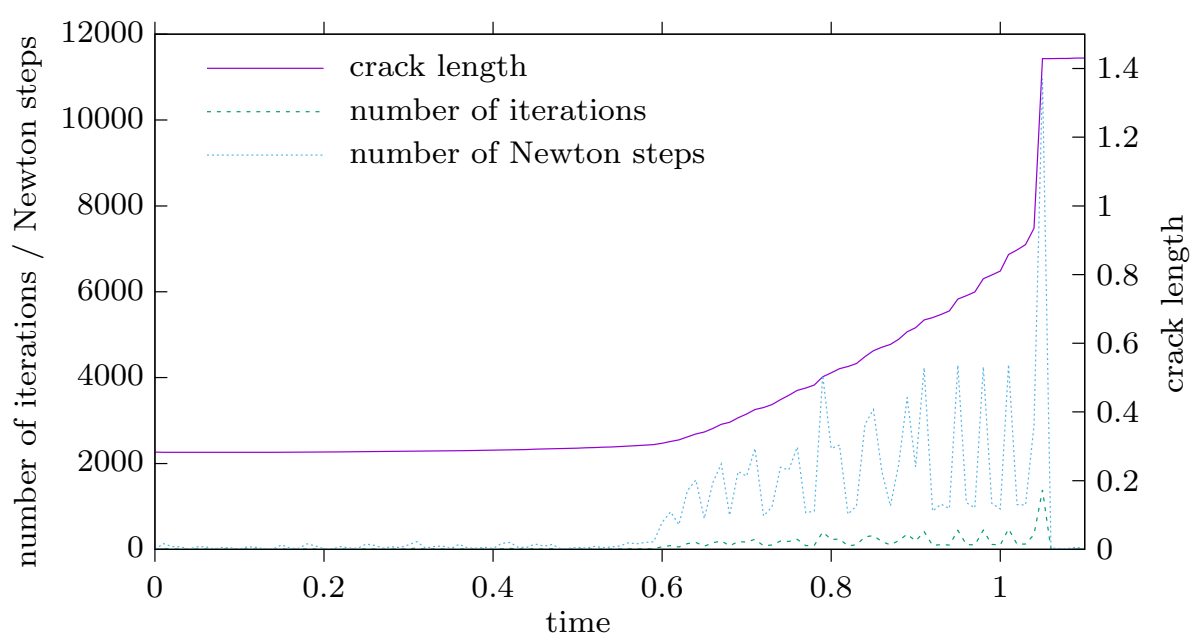

Fig. 4 Numerical output data of the second example with initial crack $\Gamma_{2}$ and boundary condition $w_{2}$

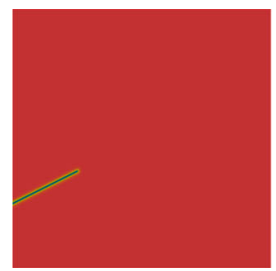

$t=0$

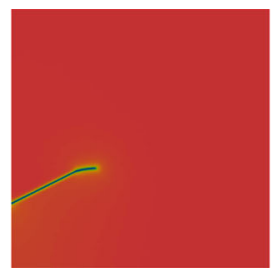

$t=0.7$

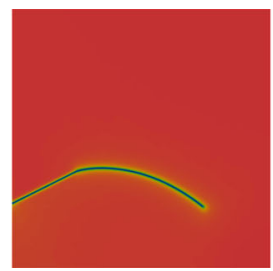

$t=1.04$

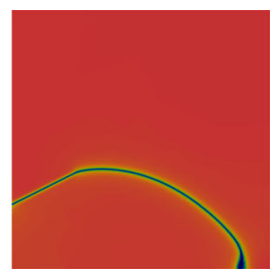

$t=1.05$

Fig. 5 Phase-field of second example at various times

Fig. 6 Mesh at time $t=1.04$

used for the first example

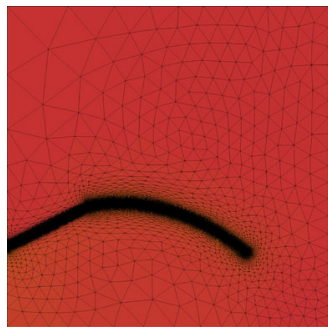

Complete mesh

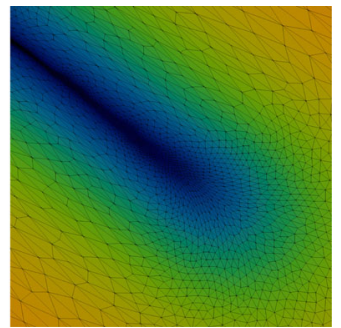

Mesh at the crack tip

analysis for $\varepsilon$ ranging from $5 \cdot 10^{-2}$ to $10^{-2}$. However, in Fig. 7 , it can be noticed that there are still considerable changes in the crack path for smaller values of $\varepsilon$.

Furthermore, we investigate the influence of the time step size. Comparing the phase fields in Fig. 7, we notice that the bending effect of the crack path reduces for decreased time step sizes. Even though the size of $\tau$ plays a much smaller role than $\varepsilon$, it can be substantial when the final part of the crack path is on the brink of turning downwards or rightwards, as it happens for $\varepsilon=2 \cdot 10^{-3}$. 


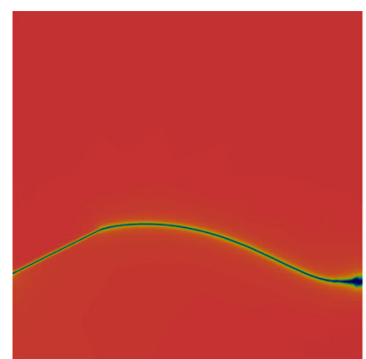

$\varepsilon=10^{-3}, \tau=10^{-2}$

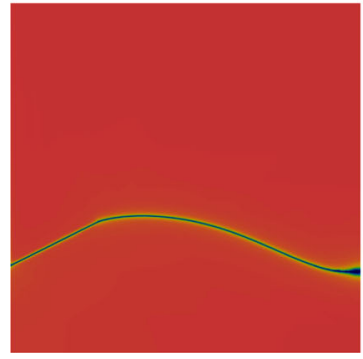

$\varepsilon=10^{-3}, \tau=10^{-3}$

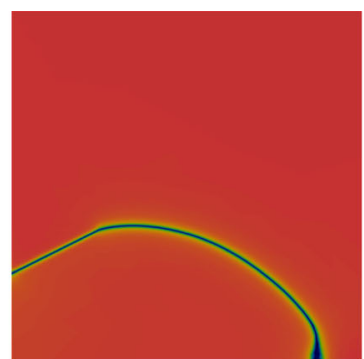

$\varepsilon=2 \cdot 10^{-3}, \tau=10^{-2}$

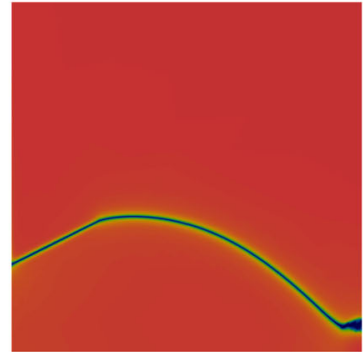

$\varepsilon=2 \cdot 10^{-3}, \tau=10^{-3}$

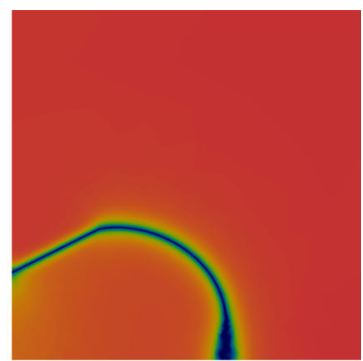

$\varepsilon=5 \cdot 10^{-3}, \tau=10^{-2}$

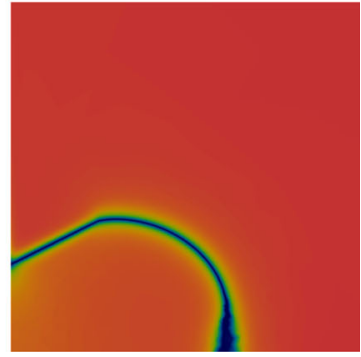

$\varepsilon=5 \cdot 10^{-3}, \tau=10^{-3}$

Fig. 7 Final phase fields in the setting of the second example for different values of $\varepsilon$ and different time step sizes $\tau$

\section{Third example}

For the last example, we again use $\Gamma_{1}$ as a pre-crack in (6.2) and $w_{1}$ as the driving boundary condition. Unlike the first example, we cut out a hole of the domain with center $\left(c_{x}, c_{y}\right):=$ $(0.8,0.25)$ and radius 0.1 . This setting makes the crack deviate from the middle line into the hole, as it can be seen in Figure 9. The last part of the crack from the border of the hole to the right boundary of the domain appears instantaneously at time $t=1.68$.

One can find a similar example in [6,18]. There the hole is equidistant from the bottom and the right boundary, and the crack completes downwards. However, from an energetically point of view, there is no clearly preferred direction due to the same resulting crack length. In order to have a predictable situation, we moved the hole closer to the right border than to the lower one. For this reason, the obtained crack path is the expected one.

As in the two previous examples, in Fig. 8, we notice the presence of two peaks in the number of iterations, coupled with a two stage behavior of the corresponding crack length. This phenomenon is related to the fact that the phase-field experiences this time two jumps: one when the fracture enters the hole and one when it exits the domain. In between, the phase-field is not significantly changing (Fig. 9).

Having a look at the meshes in Fig. 10, the diffusion of the phase-field, where it leaves the hole, goes along with an irregular mesh. This observation indicates large changes in the curvature of the phase-field where the diffusion appears and might be a consequence of the interpolation error estimate based on the Hessian of the phase-field. Using instead a suitable 


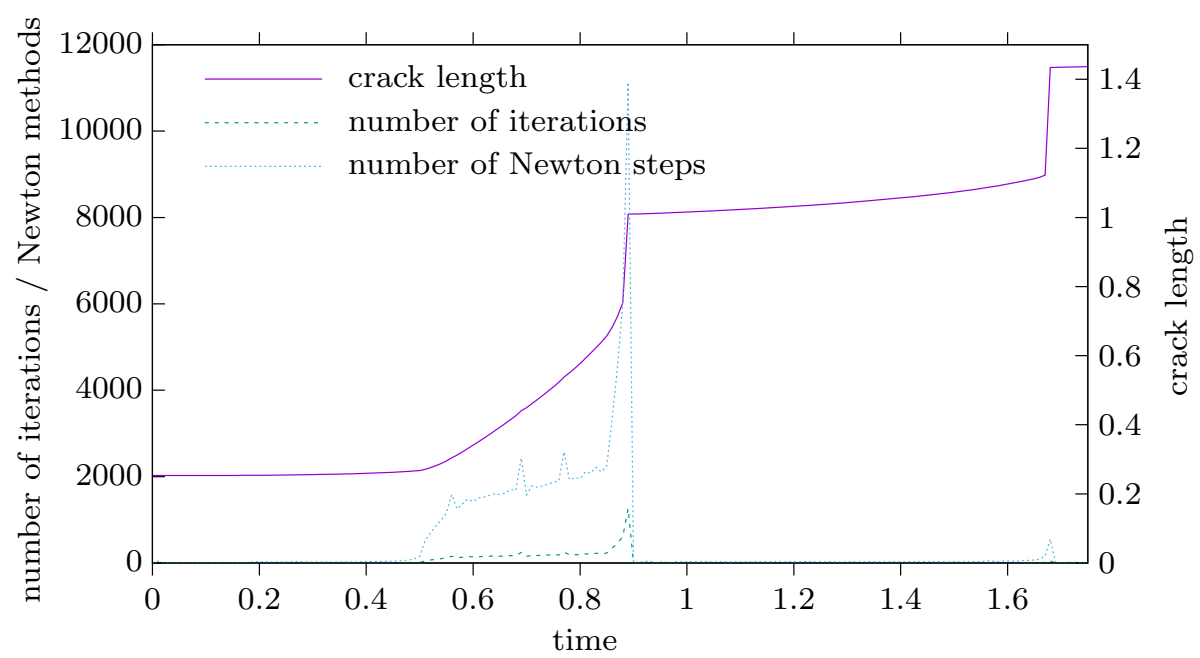

Fig. 8 Numerical output data of the third example with initial crack $\Gamma_{1}$ and boundary condition $w_{1}$ and the hole $B_{0.1}(0.8,0.25)$ cut out of the domain

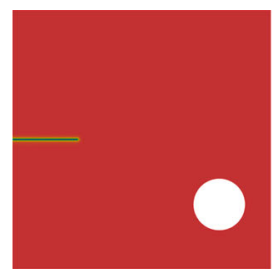

$t=0$

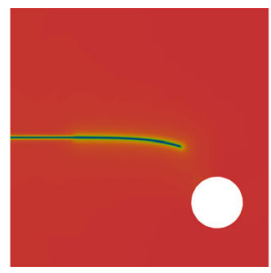

$t=0.88$

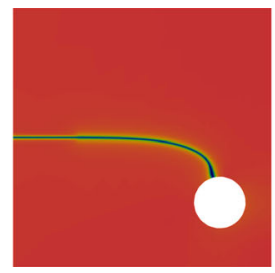

$t=0.89$

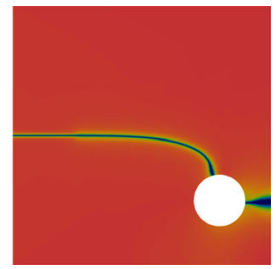

$t=1.68$

Fig. 9 Phase-field of third example at various times

Fig. 10 Mesh at time $t=0.89$

used for the first example

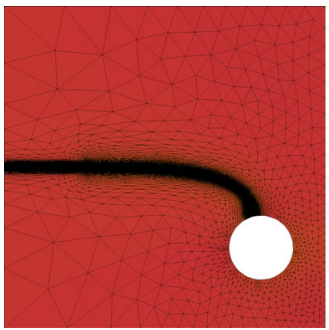

Complete mesh

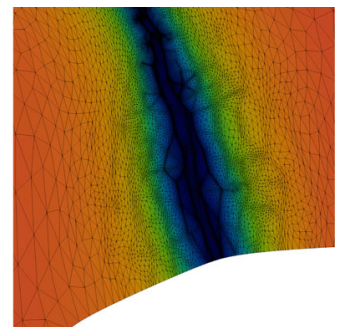

Mesh entering the hole

residual estimate as in [6] would possibly reduce this effect and should be investigated in further numerical analysis.

The computation required in total 8803 alternations with 87,602 Newton steps until the crack path was completed. It took, therefore, 96,405 linear systems to be solved. The final mesh consists of 55,050 nodes.

In Fig. 11, we continue our parameter sensitivity analysis. We not only change the value of $\varepsilon$, but also move the hole downwards as we increase $\varepsilon$. In this way, we illustrate again the sensitivity of the approximation parameter. Indeed, in the third and in the fifth plots in Fig. 11, 


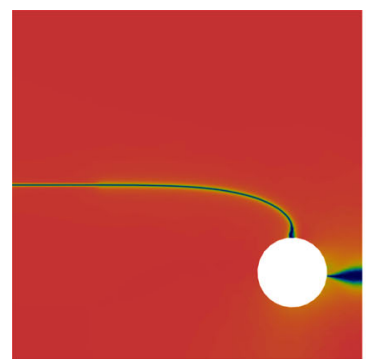

$\varepsilon=10^{-3}, c_{y}=0.25$

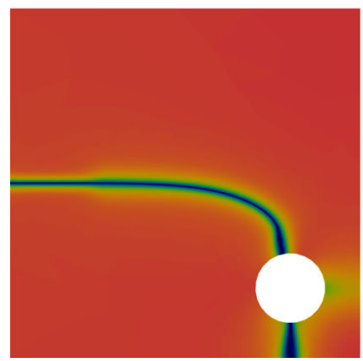

$\varepsilon=5 \cdot 10^{-3}, c_{y}=0.2$

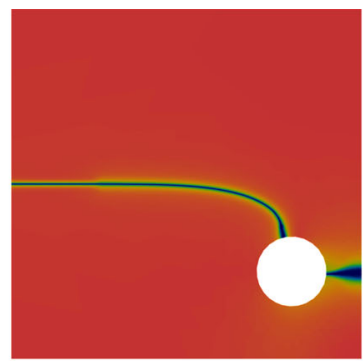

$\varepsilon=2 \cdot 10^{-3}, c_{y}=0.25$

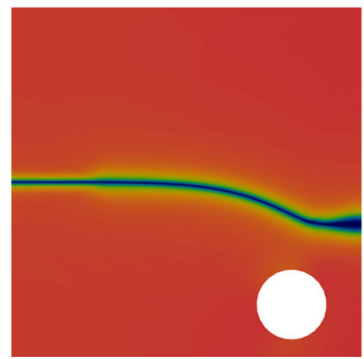

$\varepsilon=5 \cdot 10^{-3}, c_{y}=0.15$

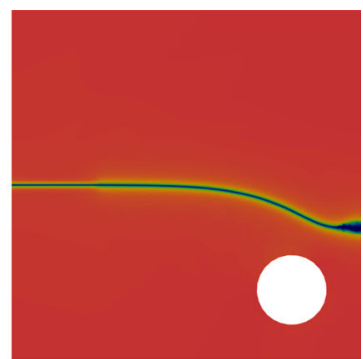

$\varepsilon=2 \cdot 10^{-3}, c_{y}=0.2$

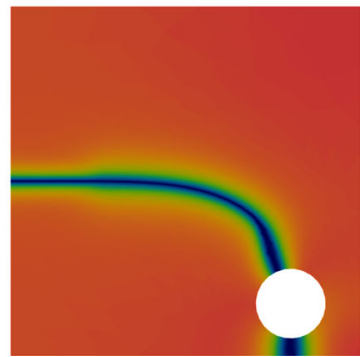

$\varepsilon=10^{-2}, c_{y}=0.15$

Fig. 11 Final phase fields in the setting of the third example for different values of $\varepsilon$ and $c_{y}$

we observe that very little movement of the hole can be sufficient to make the crack missing it. In the fourth and in the sixth plots, instead, we notice that by increasing $\varepsilon$ the crack path crosses again the hole. These phenomena are also in this case due to the interaction between the crack set and the boundary of the domain, which is modulated by the size parameter $\varepsilon$. Due to the different sizes of the domain, the simulations in $[5,18]$ are comparable to ours with $\varepsilon=10^{-2}$. As already mentioned for the second example, also in Fig. 11, we clearly observe significant variations of the crack path for values of $\varepsilon$ smaller than $10^{-2}$.

Acknowledgements We thank M. Fornasier for suggesting the research direction within the scope of the DFG Project P7 "Identification of Energies from Observations of Evolutions" of SPP 1962. S.A. acknowledges the support of SFB Transregio 109 through the project C03 "Shearlet approximation of brittle fracture evolutions". S.B. acknowledges the support of DFG through the International Research Training Group IGDK 1754 "Optimization and Numerical Analysis for Partial Differential Equations with Nonsmooth Structures".

\section{Appendix A}

Lemma A.1 Let $h>0$. For every $M>0$, there exists a positive constant $C=C(M)$ such that for every $u, v \in \mathcal{F}_{h}$ with $\|\nabla u\|_{\infty},\|v\|_{\infty} \leq M$, for every $\varphi \in \mathcal{A}_{h}$, and every $\psi \in \mathcal{F}_{h}$ we have

$$
\frac{1}{C}\|\varphi\|_{H^{1}} \leq\|\varphi\|_{h, v} \leq C\|\varphi\|_{H^{1}} \quad \frac{1}{C}\|\psi\|_{H^{1}} \leq\|\psi\|_{h, u} \leq C\|\psi\|_{H^{1}} .
$$

Proof The proof follows directly from the definition (2.27)-(2.28) of the two norms. 
Lemma A.2 Let $h>0$. Let $w_{j}, v_{j} \in \mathcal{F}_{h}$ and let $u_{j} \in \mathcal{A}_{h}\left(w_{j}\right)$. Assume that there exist $w, v \in \mathcal{F}_{h}$ and $u \in \mathcal{A}_{h}(w)$ such that $w_{j} \rightarrow w, v_{j} \rightarrow v$, and $u_{j} \rightarrow u$ in $\mathcal{F}_{h}$ as $j \rightarrow+\infty$. Then

$$
\left|\partial_{u} \mathcal{J}_{h}\right|(u, v)=\lim _{j}\left|\partial_{u} \mathcal{J}_{h}\right|\left(u_{j}, v_{j}\right) \quad\left|\partial_{v} \mathcal{J}_{h}\right|(u, v)=\lim _{j}\left|\partial_{v} \mathcal{J}_{h}\right|\left(u_{j}, v_{j}\right) .
$$

Proof Fix $\varphi \in \mathcal{A}_{h}(0)$ with $\|\varphi\|_{h, v} \leq 1$. Since $\|\varphi\|_{h, v_{j}} \rightarrow\|\varphi\|_{h, v}$ as $j \rightarrow+\infty$, we consider the following function:

$$
\varphi_{j}:= \begin{cases}\varphi & \text { if }\|\varphi\|_{h, v}<1, \\ \frac{\varphi}{\|\varphi\|_{h, v_{j}}} & \text { if }\|\varphi\|_{h, v}=1 .\end{cases}
$$

Clearly $\varphi_{j} \rightarrow \varphi$ in $\mathcal{F}_{h}$ as $j \rightarrow+\infty$. Moreover, by definition (2.29) of the slope w.r.t. the displacement $u$, we have that

$$
\liminf _{j}\left|\partial_{u} \mathcal{J}_{h}\right|\left(u_{j}, v_{j}\right) \geq-\lim _{j} \partial_{u} \mathcal{J}_{h}\left(u_{j}, v_{j}\right)\left[\varphi_{j}\right]=-\partial_{u} \mathcal{J}_{h}(u, v)[\varphi] .
$$

Hence, we deduce that $\left|\partial_{u} \mathcal{J}_{h}\right|(u, v) \leq \liminf _{j}\left|\partial_{u} \mathcal{J}_{h}\right|\left(u_{j}, v_{j}\right)$.

For the opposite inequality, let $\varphi_{j} \in \mathcal{A}_{h}(0)$ be such that

$$
\left|\partial_{u} \mathcal{J}_{h}\right|\left(u_{j}, v_{j}\right)=-\partial_{u} \mathcal{J}_{h}\left(u_{j}, v_{j}\right)\left[\varphi_{j}\right] .
$$

Up to a subsequence, we may assume that $\varphi_{j} \rightarrow \varphi \in \mathcal{A}_{h}(0)$. Hence, $\left\|\varphi_{j}\right\|_{h, v_{j}} \rightarrow\|\varphi\|_{h, v} \leq 1$ and

$$
\limsup _{j}\left|\partial_{u} \mathcal{J}_{h}\right|\left(u_{j}, v_{j}\right)=-\lim _{j} \partial_{u} \mathcal{J}_{h}\left(u_{j}, v_{j}\right)\left[\varphi_{j}\right]=-\partial_{u} \mathcal{J}_{h}(u, v)[\varphi] \leq\left|\partial_{u} \mathcal{J}_{h}\right|(u, v),
$$

which conclude the proof of the first equality in (A.1). In a similar way we can prove the second.

Proof of Proposition 3.1 It is enough to prove the statement for $j=1$. In order to show the existence of a minimizer for (3.2), we want to apply the direct method of the calculus of variations. Since $\mathcal{J}_{h}\left(u_{1}, \cdot\right)$ is continuous with respect to the convergence in $\mathcal{F}_{h}$, we only need to show that a minimizing sequence $z_{k} \in \mathcal{F}_{h}$ for (3.2) admits a limit, at least up to a subsequence. Since

$$
\sup _{k \in \mathbb{N}}\left\|\nabla z_{k}\right\|_{2}^{2} \leq \sup _{k \in \mathbb{N}} 2 \mathcal{J}_{h}\left(u_{1}, z_{k}\right)<+\infty,
$$

by construction of the function space $\mathcal{F}_{h}(2.15)$ and of the triangulation $\mathcal{T}_{h}$, we easily deduce that

$$
\sup _{k}\left\|\nabla z_{k}\right\|_{\infty}<+\infty,
$$

so that the functions $z_{k}$ are uniformly Lipschitz in $\Omega$. Moreover, since by hypothesis $v_{0} \geq 0$, it is not restrictive to assume that there exists at least one vertex $x_{l}, l \in\left\{1, \ldots, N_{h}\right\}$, such that $z_{k}\left(x_{l}\right) \geq 0$. Indeed, if $z_{k}<0$ in $\Omega$, it is readily seen that $\mathcal{J}_{h}\left(u_{1}, 0\right) \leq \mathcal{J}_{h}\left(u_{1}, z_{k}\right)$. Therefore, thanks to (A.2) and to the inequality $z_{k} \leq v_{0}$ in $\Omega$, we also deduce that the sequence $z_{k}$ is uniformly bounded in $W^{1, \infty}(\Omega)$. Thus, $z_{k}$ converges, up to a subsequence, to some $v_{1}$ in $\mathcal{F}_{h}$, and this concludes the proof of existence. The uniqueness of solution follows by the strict convexity of the functional $\mathcal{J}_{h}\left(u_{1}, \cdot\right)$.

We now prove the second part of the statement, i.e., that $0 \leq v_{1} \leq 1$. For the sake of contradiction, let us first assume that $v_{1} \ngtr 0$. Using the notation described in Sect. 2, let $x_{l} \in \Delta_{h}$ be such that $v_{1}\left(x_{l}\right) \leq v_{1}\left(x_{m}\right)$ for every $m=1, \ldots, N_{h}$. In particular, we 
have $v_{1}\left(x_{l}\right)<0$. Let $\xi_{l} \geq 0$ be the $l$-th element of the basis of $\mathcal{F}_{h}$ defined by (2.16). Being $v_{0} \geq 0$ in $\Omega$ and $v_{1}\left(x_{l}\right)<0$, for every $\varepsilon \in \mathbb{R}$ with $|\varepsilon|$ small enough we have that $v_{1}+\varepsilon \xi_{l} \leq v_{0}$ in $\Omega$, and, by the minimality of $v_{1}, \mathcal{J}_{h}\left(u_{1}, v_{1}\right) \leq \mathcal{J}_{h}\left(u_{1}, v_{1}+\varepsilon \xi_{l}\right)$. By the quadratic structure of $\mathcal{J}_{h}\left(u_{1}, \cdot\right)$, from the previous inequality and the arbitrariness of $\varepsilon$, we deduce that

$$
\int_{\Omega} \mathrm{P}_{h}\left(v_{1} \xi_{l}\right)\left|\nabla u_{1}\right|^{2} \mathrm{~d} x+\int_{\Omega} \nabla v_{1} \cdot \nabla \xi_{l} \mathrm{~d} x-\int_{\Omega} \mathrm{P}_{h}\left(\left(1-v_{1}\right) \xi_{l}\right) \mathrm{d} x=0 .
$$

Since $v_{1}\left(x_{l}\right)<0$ and (2.16) holds, we have that

$$
\mathrm{P}_{h}\left(v_{1} \xi_{l}\right) \leq 0 \text { and } \mathrm{P}_{h}\left(\left(1-v_{1}\right) \xi_{l}\right) \geq 0 \quad \text { in } \Omega \text {. }
$$

Hence, from (A.3) and (A.4) we get

$$
\int_{\Omega} \nabla v_{1} \cdot \nabla \xi_{l} \mathrm{~d} x=-\int_{\Omega} \mathrm{P}_{h}\left(v_{1} \xi_{l}\right)\left|\nabla u_{1}\right|^{2} \mathrm{~d} x+\int_{\Omega} \mathrm{P}_{h}\left(\left(1-v_{1}\right) \xi_{l}\right) \mathrm{d} x \geq 0 .
$$

On the other hand, we can write $v_{1}$ as a linear combination of the elements of the basis $\left\{\xi_{m}\right\}_{m=1}^{N_{h}}$ of $\mathcal{F}_{h}$, namely, $v_{1}=\sum_{m=1}^{N_{h}} v_{1}\left(x_{m}\right) \xi_{m}$. Then, by direct computation,

$$
\begin{aligned}
& \int_{\Omega} \nabla v_{1} \cdot \nabla \xi_{l} \mathrm{~d} x=\sum_{m=1}^{N_{h}} v_{1}\left(x_{m}\right) \int_{\Omega} \nabla \xi_{m} \cdot \nabla \xi_{l} \mathrm{~d} x \\
& =v_{1}\left(x_{l}\right) \sum_{m=1}^{N_{h}} \int_{\Omega} \nabla \xi_{m} \cdot \nabla \xi_{l} \mathrm{~d} x+\sum_{m=1}^{N_{h}}\left(v_{1}\left(x_{m}\right)-v_{1}\left(x_{l}\right)\right) \int_{\Omega} \nabla \xi_{m} \cdot \nabla \xi_{l} \mathrm{~d} x \\
& =\sum_{m=1}^{N_{h}}\left(v_{1}\left(x_{m}\right)-v_{1}\left(x_{l}\right)\right) \int_{\Omega} \nabla \xi_{m} \cdot \nabla \xi_{l} \mathrm{~d} x \leq 0
\end{aligned}
$$

where, in the last equality, we have used (2.17), the particular choice of the vertex $x_{l}$, and the fact that

$$
\sum_{m=1}^{N_{h}} \int_{\Omega} \nabla \xi_{m} \cdot \nabla \xi_{l} \mathrm{~d} x=0
$$

Therefore, combining (A.5) and (A.6), we get a contradiction, and thus $v_{1} \geq 0$.

In order to show that $v_{1} \leq 1$, we can argue again by contradiction, assuming that there exists a vertex $x_{l}, l \in\left\{1, \ldots, N_{h}\right\}$ such that $v_{1}\left(x_{l}\right) \geq v_{1}\left(x_{m}\right)$ for every $m=1, \ldots, N_{h}$ and $v_{1}\left(x_{l}\right)>1$. As before, being $\xi_{l}$ the $l$-th element of the basis of $\mathcal{F}_{h}$, for $\varepsilon \in \mathbb{R}$ with $|\varepsilon|$ small enough there holds $v_{1}+\varepsilon \xi_{l} \geq v_{0}$, such that we can repeat (A.3)-(A.6) with opposite inequality signs, concluding that $0 \leq v_{1} \leq 1$ in $\Omega$. This concludes the proof of the proposition.

Proof of Lemma 5.1 Let $M>0, g \in C^{2}(\mathbb{R})$, the mesh parameter $h>0$, and the triangulation $\mathcal{T}_{h}$ be fixed, and let us consider a function $v \in \mathcal{F}_{h}$ such that $\|v\|_{\infty} \leq M$.

Given an element $K$ of the triangulation $\mathcal{T}_{h}$, we first prove (5.1) on $K$. Without loss of generality, we may assume that the origin is a vertex of $K$ and

$$
K=\left\{x=\left(x_{1}, x_{2}\right) \in \Omega: x_{1} \in[0, h], x_{2} \in\left[0, x_{1}\right]\right\} .
$$

The general case follows by an affine transformation.

Recalling that if a function $v \in \mathcal{F}_{h}$, then it is affine on every element of $\mathcal{T}_{h}$, for every $x \in K$ we have

$$
v(x)=v(0)+\left.\nabla v\right|_{K} \cdot x
$$


Hence, by Taylor expansion, there exists some $\xi \in K$ such that

$$
g(v(x))=g(v(0))+g^{\prime}(v(0))(v(x)-v(0))+\frac{1}{2} g^{\prime \prime}(v(\xi))(v(x)-v(0))^{2} .
$$

In particular, the last term in (A.7) can be simply estimated by

$$
\left|g^{\prime \prime}(v(\xi))\right|(v(x)-v(0))^{2} \leq\left.\left|g^{\prime \prime}(v(\xi))\right||\nabla v|_{K}\right|^{2}|x|^{2} .
$$

Recalling the definition of $\mathrm{P}_{h}$ (2.19) and using the expansion (A.7) we can write

$$
\begin{aligned}
\mathrm{P}_{h}(g \circ v)(x)= & g(v(0))+\frac{1}{h}(g(v(h, 0))-g(v(0))) x_{1} \\
& +\frac{1}{h}(g(v(0, h))-g(v(0))) x_{2} \\
= & g(v(0))+\frac{1}{h} g^{\prime}(v(0))\left((v(h, 0)-v(0)) x_{1}+(v(0, h)-v(0)) x_{2}\right) \\
& +\frac{1}{2 h} g^{\prime \prime}\left(v\left(\xi_{1}\right)\right)|v(h, 0)-v(0)|^{2} x_{1} \\
& +\frac{1}{2 h} g^{\prime \prime}\left(v\left(\xi_{2}\right)\right)|v(0, h)-v(0)|^{2} x_{2}
\end{aligned}
$$

for some $\xi_{1}, \xi_{2} \in K$. Hence, in view of (A.7)-(A.9), for $x \in K$ there holds

$$
\left|g(v(x))-\mathrm{P}_{h}(g \circ v)(x)\right| \leq\left. C h^{2}|\nabla v|_{K}\right|^{2}
$$

for some positive constant $C=C(g, M)$ depending only on $g \in C^{2}(\mathbb{R})$ and on $M$. Integrating the previous inequality, we end up with (5.1), and the proof is concluded.

\section{References}

1. Ambrosio, L., Fusco, N., Pallara, D.: Functions of Bounded Variation and Free Discontinuity Problems. Oxford Mathematical Monographs. The Clarendon Press, New York (2000)

2. Ambrosio, L., Tortorelli, V.M.: Approximation of functionals depending on jumps by elliptic functionals via $\Gamma$-convergence. Commun. Pure Appl. Math. 43, 999-1036 (1990)

3. Ambrosio, L., Tortorelli, V.M.: On the approximation of free discontinuity problems. Boll. Un. Mat. Ital. B (7) 6, 105-123 (1992)

4. Artina, M., Cagnetti, F., Fornasier, M., Solombrino, F.: Linearly constrained evolutions of critical points and an application to cohesive fractures. Math. Models Methods Appl. Sci. 27, 231-290 (2017)

5. Artina, M., Fornasier, M., Micheletti, S., Perotto, S.: Anisotropic adaptive meshes for brittle fractures: parameter sensitivity. In: Numerical Mathematics and Advanced Applications-ENUMATH 2013, vol. 103 of Lecturer Notes in Computer Science Engineering, pp. 293-301. Springer, Cham (2015)

6. Artina, M., Fornasier, M., Micheletti, S., Perotto, S.: Anisotropic mesh adaptation for crack detection in brittle materials. SIAM J. Sci. Comput. 37, B633-B659 (2015)

7. Artina, M., Fornasier, M., Micheletti, S., Perotto, S.: The benefits of anisotropic mesh adaptation for brittle fractures under plane-strain conditions. In: New Challenges in Grid Generation and Adaptivity for Scientific Computing, vol. 5 of SEMA SIMAI Springer Series, pp. 43-67. Springer, Cham (2015)

8. Balder, E.J.: An extension of Prohorov's theorem for transition probabilities with applications to infinitedimensional lower closure problems. Rend. Circ. Mat. Palermo (2) 34(1985), 427-447 (1986)

9. Bertsekas, D.P.: Projected Newton methods for optimization problems with simple constraints. SIAM J. Control Optim. 20, 221-246 (1982)

10. Bourdin, B.: Numerical implementation of the variational formulation for quasi-static brittle fracture. Interfaces Free Bound. 9, 411-430 (2007)

11. Bourdin, B.: The variational formulation of brittle fracture: numerical implementation and extensions. In: Combescure, A., De Borstiutam, R. (eds.) Symposium on Discretization Methods for Evolving Discontinuities, vol. 5 of IUTAM Bookseries, p. 381. Springer, Dordrecht (2007) 
12. Bourdin, B., Chambolle, A.: Implementation of an adaptive finite-element approximation of the MumfordShah functional. Numer. Math. 85, 609-646 (2000)

13. Bourdin, B., Francfort, G.A., Marigo, J.-J.: Numerical experiments in revisited brittle fracture. J. Mech. Phys. Solids 48, 797-826 (2000)

14. Bourdin, B., Francfort, G.A., Marigo, J.-J.: The variational approach to fracture. J. Elast. 91, 5-148 (2008)

15. Braides, A.: Approximation of Free-Discontinuity Problems, vol. 1694 of Lecture Notes in Mathematics. Springer, Berlin (1998)

16. Braides, A., Larsen, C.J.: $\Gamma$-convergence for stable states and local minimizers. Ann. Sc. Norm. Super. Pisa Cl. Sci. (5) 10, 193-206 (2011)

17. Braides, A., Truskinovsky, L.: Asymptotic expansions by $\Gamma$-convergence. Contin. Mech. Thermodyn. 20, 21-62 (2008)

18. Burke, S., Ortner, C., Süli, E.: An adaptive finite element approximation of a variational model of brittle fracture. SIAM J. Numer. Anal. 48, 980-1012 (2010)

19. Burke, S., Ortner, C., Süli, E.: Adaptive finite element approximation of the Francfort-Marigo model of brittle fracture. In: Approximation and computation, vol. 42 of Springer optimization and Applications, pp. 297-310. Springer, New York (2011)

20. Burke, S., Ortner, C., Süli, E.: An adaptive finite element approximation of a generalized AmbrosioTortorelli functional. Math. Models Methods Appl. Sci. 23, 1663-1697 (2013)

21. Chambolle, A.: Image segmentation by variational methods: Mumford and Shah functional and the discrete approximations. SIAM J. Appl. Math. 55, 827-863 (1995)

22. Chambolle, A., Dal Maso, G.: Discrete approximation of the Mumford-Shah functional in dimension two. M2AN Math. Model. Numer. Anal. 33, 651-672 (1999)

23. Ciarlet, P.G., Raviart, P.-A.: Maximum principle and uniform convergence for the finite element method. Comput. Methods Appl. Mech. Eng. 2, 17-31 (1973)

24. Fleischer, I., Porter, J.E.: Convergence of metric space-valued BV functions. Real Anal. Exchange 27,315$319(2001 / 02)$

25. Fonseca, I., Leoni, G.: Modern Methods in the Calculus of Variations: $L^{p}$ spaces. Springer Monographs in Mathematics. Springer, New York (2007)

26. Francfort, G.A., Marigo, J.-J.: Revisiting brittle fracture as an energy minimization problem. J. Mech. Phys. Solids 46, 1319-1342 (1998)

27. Giacomini, A.: Ambrosio-Tortorelli approximation of quasi-static evolution of brittle fractures. Calc. Var. Part. Differ. Equ. 22, 129-172 (2005)

28. Giacomini, A., Ponsiglione, M.: A discontinuous finite element approximation of quasi-static growth of brittle fractures. Numer. Funct. Anal. Optim. 24, 813-850 (2003)

29. Giacomini, A., Ponsiglione, M.: Discontinuous finite element approximation of quasistatic crack growth in nonlinear elasticity. Math. Models Methods Appl. Sci. 16, 77-118 (2006)

30. Hecht, F.: New development in freefem++. J. Numer. Math. 20, 251-265 (2012)

31. Kelley, C.T.: Iterative Methods for Optimization, vol. 18 of Frontiers in Applied Mathematics. Society for Industrial and Applied Mathematics (SIAM), Philadelphia (1999)

32. Knees, D., Negri, M.: Convergence of alternate minimization schemes for phase-field fracture and damage. Math. Models Methods Appl. Sci. 27, 1743-1794 (2017)

33. Mielke, A.: Evolution of rate-independent systems. In: Evolutionary Equations. vol. II, Handbook of Differential Equations, pp. 461-559. Elsevier/North-Holland, Amsterdam (2005)

34. Mielke, A., Roubicek, T.: Rate-independent systems, vol. 193 of Applied Mathematical Sciences, Theory and application. Springer, New York (2015)

35. Mielke, A., Roubicek, T., Stefanelli, U.: $\Gamma$-limits and relaxations for rate-independent evolutionary problems. Calc. Var. Part. Differ. Equ. 31, 387-416 (2008)

36. Negri, M.: Quasi-static rate-independent evolutions: characterization, existence, approximation and application to fracture mechanics. ESAIM Control Optim. Calc. Var. 20, 983-1008 (2014)

37. Negri, M., Almi, S., Belz, S.: Convergence of discrete and continuous unilateral flows for AmbrosioTortorelli energies and application to mechanics. ESAIM M2AN (2018). https://doi.org/10.1051/m2an/ 2018057

38. Quarteroni, A., Valli, A.: Numerical Approximation of Partial Differential Equations. vol. 23 of Springer Series in Computational Mathematics. Springer, Berlin (1994)

Publisher's Note Springer Nature remains neutral with regard to jurisdictional claims in published maps and institutional affiliations. 